\title{
COLLOQUIUM LECTURES ON GEOMETRIC MEASURE THEORY ${ }^{1}$
}

\author{
BY HERBERT FEDERER
}

1. Introduction. The early discoveries of measure theory, at the start of this century, led to a very good understanding of how subsets of Euclidean $n$ space

$\mathbf{R}^{n}$
behave with respect to $n$ dimensional Lebesgue measure

$e^{n}$.

Much of the theory of functions was revolutionized by Lebesgue's method of integration. This paved the way for great advances in Fourier analysis. Furthermore Lebesgue's contributions to measure theory made possible the application of direct methods in the one dimensional calculus of variations, which soon developed to a highly satisfactory stage.

Many two dimensional variational problems, including some versions of the problem of Plateau, have also been solved through ingenious extension of Lebesgue's methods, making use of conformal parametrizations and Dirichlet integrals. However, these classical methods have failed to give significant results for the part of the calculus of variations involving parametric integrals over $m$ dimensional surfaces in case $m$ exceeds two. Concrete examples show that sets representing solutions of high dimensional variational problems can have very complicated singularities. To account for geometric actuality, and in order to prove general existence theorems, one must abandon the idea of describing all the competing surfaces by continuous maps from a single predetermined parameter space. One should rather think of surfaces as $m$ dimensional mass distributions, with tangent $m$ vectors attached. Then the boundary conditions must be expressed in a manner quite different from functional restriction-for instance through a boundary operator as in algebraic topology.

It took five decades, beginning with Carathéodory's fundamental paper on measure theory in 1914, to develop the intuitive conception of an $m$ dimensional surface as a mass distribution into an efficient instrument of mathematical analysis, capable of significant applications in the calculus of variations. The first three decades were spent learning basic facts on how subsets of $\mathbf{R}^{n}$ behave with respect to $m$ dimensional Hausdorff measure

$$
\mathcal{H}^{m} \text {. }
$$

Colloquium Lectures given at the Summer Meeting of the American Mathematical Society on August 15-18, 1977 in Seattle, Washington; received by the editors August 25, 1977.

AMS (MOS) subject classifications (1970). Primary 49F20, 49F22; Secondary 26A45, 26A57, 26A63, 26A66, 28A75, 42A92, 49F05, 49F10, 49F25, 53C65.

'This work was supported in part by grants from the John Simon Guggenheim Memorial Foundation and the National Science Foundation. 
During the next two decades this knowledge was fused with many techniques from analysis, geometry and algebraic topology, finally to produce new and sometimes surprising but classically acceptable solutions to old problems.

Geometric measure theory developed in its formative period mainly through the research of mathematicians working on the following topics:

Rectifiability, density and projection properties of sets with finite $m$ dimensional Hausdorff measure (Gross, Besicovitch, Randolph, A. P. Morse, Federer, Freilich, E. F. Moore, Marstrand, Mickle),

Lebesgue area and Plateau's problem (De Geöcze, Tonelli, Radó, Douglas, Morrey, McShane, Cesari, Federer, Besicovitch, Reifenberg, Tompson, Demers, Goffman),

Gauss-Green theorem and sets with finite perimeter (Federer, De Giorgi),

Functions whose distribution derivatives are representable by integration (Tonelli, Cesari, Federer, Krickeberg, Fleming),

Generalized surfaces, alias varifolds (L. C. Young, Fleming),

Differential forms and currents (De Rham),

Flat chains with real coefficients (Whitney),

Normal currents, integral currents and flat chains with discrete coefficients (Fleming, Federer),

Partial regularity of measure theoretic solutions of Plateau's problem (Fleming, De Giorgi, Reifenberg).

I believe that by 1964, fifty years after the publication of Carathéodory's paper, geometric measure theory had proved its worth beyond a reasonable doubt. In the ensuing period the subject has grown steadily, contributing significantly to other parts of mathematics, and has been enriched by the research of a new generation (W. K. Allard, F. J. Almgren, E. Bombieri, K. E. Brakke, J. E. Brothers, A. Chiffi, L. R. Ernst, A. T. Fomenko, R. Gariepy, E. Giusti, R. M. Hardt, R. Harvey, S. Kar, J. R. King, R. V. Kohn, J. Kral, H. B. Lawson, P. Mattila, J. H. Michael, M. Miranda, F. Morgan, H. R. Parks, S. Paur, J. T. Pitts, E. Santi, V. Scheffer, R. Schoen, B. Shiffman, L. Simon, J. Simons, J. E. Taylor, D. Triscari, A. I. Volpert, W. P. Ziemer).

Much more remains to be done than has been done, and the important unsolved problems appear extremely difficult, but hopefully we now have an adequate basic structure to support and direct our future efforts.

In these lectures I shall give an exposition, without proofs, of some of the principal concepts and results of geometric measure theory. More extensive and detailed information on the subject is available in my book published in 1969, and in subsequent papers listed in the appended supplementary bibliography.

2. Hausdorff measure and rectifiability. For every nonnegative real number $m$ we define

$$
\alpha(m)=\Gamma(1 / 2)^{m} / \Gamma(m / 2+1) .
$$

Whenever $S \subset \mathbf{R}^{n}$ and $\delta>0$ we let

$$
\mathcal{H}^{m, \delta}(S)
$$

be the infimum of the set of numbers 


$$
\sum_{T \in G} \boldsymbol{\alpha}(m)[\operatorname{diam}(T) / 2]^{m}
$$

corresponding to all countable coverings $G$ of $S$ with

$$
\operatorname{diam}(T) \leqslant \delta \text { for } T \in G \text {; }
$$

then we define the $m$ dimensional Hausdorff measure

$$
\mathcal{H}^{m}(S)=\lim _{\delta \downarrow 0} \mathcal{H}^{m, \delta}(S)
$$

We also define the

$$
\text { Hausdorff dimension of } S=\inf \left\{m: \mathcal{H}^{m}(S)=0\right\} \text {. }
$$

One finds that

$$
\mathcal{H}^{0}(S)=\text { the number of elements of } S, \quad \mathcal{H}^{n}(S)=\mathfrak{L}^{n}(S),
$$

and that every number between 0 and $n$ occurs as Hausdorff dimension of some compact subset of $\mathbf{R}^{n}$; for example the classical Cantor set has Hausdorff dimension $\log (2) / \log (3)$ [23, \$2.10.28].

The (outer) measure $\mathcal{F C}^{m}$ over $\mathbf{R}^{n}$ is Borel regular.

With each measure $\gamma$ over $\mathbf{R}^{n}$ and each subset $W$ of $\mathbf{R}^{n}$ we associate the measure $\gamma\llcorner W$ such that

$$
\left(\gamma\llcorner W)(S)=\gamma(W \cap S) \text { for } S \subset \mathbf{R}^{n} .\right.
$$

If $\gamma$ is Borel regular, so is $\gamma L W$; if also

$$
\gamma(W \cap K)<\infty \text { for every compact } K \subset \mathbf{R}^{n},
$$

then $\gamma L W$ is a Radon measure.

Important local attributes of a measure are its densities and its approximate tangent cones. To describe these concepts we will use the closed balls

$$
\mathbf{B}(a, r)=\{x:|x-a| \leqslant r\}
$$

with center $a$ and radius $r$, as well as the open cones

$$
\mathbf{E}(a, v, \varepsilon)=\{x:|t(x-a)-v|<\varepsilon \text { for some } t>0\}
$$

with vertex $a$ and axis in the direction $v$, whose generating rays from $a$ come within distance $\varepsilon$ of $a+v$. We define the upper and lower $m$ dimensional densities of the measure $\gamma$ at the point $a$ as

$$
\Theta^{* m}(\gamma, a)=\underset{r \downarrow 0}{\lim \sup } \gamma[\mathbf{B}(a, r)] /\left[\boldsymbol{\alpha}(m) r^{m}\right]
$$

and

$$
\Theta_{*}^{m}(\gamma, a)=\liminf _{r \downarrow 0} \gamma[\mathbf{B}(a, r)] /\left[\boldsymbol{\alpha}(m) r^{m}\right] .
$$

In case the upper and lower densities are equal, their common value is the $m$ dimensional density

$$
\Theta^{m}(\gamma, a)
$$

Densities have considerable intrinsic interest in measure theory and elsewhere, for example as multiplicities of holomorphic varieties. Right now we merely call attention to their role in comparing any Radon measure $\gamma$ to 
$\mathcal{H}^{m}$ on a subset $S$ of $\mathbf{R}^{n}[23, \S 2.10 .19]$ :

If $\Theta^{* m}(\gamma, a)<t$ for $a \in S$, then $\gamma(S) \leqslant 2^{m} t \mathcal{H}^{m}(S)$.

If $\Theta^{* m}(\gamma, a)>t$ for $a \in S$, then $\gamma(S) \geqslant t \mathcal{H}^{m}(S)$.

Moreover, in case $S$ is $\gamma$ measurable, then

$$
\Theta^{m}\left(\gamma\llcorner S, x)=0 \text { for } \mathcal{H}^{m} \text { almost all } x \text { in } \mathbf{R}^{n} \sim S .\right.
$$

Taking $\gamma=\mathscr{H}^{m}\left\llcorner W\right.$, where $\mathfrak{H}^{m}(W)<\infty$, we infer

$$
\begin{gathered}
\Theta^{* m}\left(\mathcal{H}^{m} L W, a\right)>2^{-m} \text { for } \mathcal{H}^{m} \text { almost all } a \text { in } W, \\
\Theta^{* m}\left(\mathcal{H}^{m} L W, a\right) \leqslant 1 \text { for } \mathcal{H}^{m} \text { almost all } a .
\end{gathered}
$$

However, it can happen that $\Theta_{*}^{m}\left(\mathcal{H}^{m} L W, a\right)=0$ for all $a$, even though $\mathcal{F}^{m}(W)>0$. Accordingly upper densities are generally more useful than lower densities.

For any measure $\gamma$ over $\mathbf{R}^{n}$ we define the $(\gamma, m)$ approximate tangent vectors at the point $a$ as the elements of the closed cone (with vertex 0 )

$$
\operatorname{Tan}^{m}(\gamma, a)=\bigcap_{\varepsilon>0}\left\{v: \Theta^{* m}[\gamma\llcorner\mathbf{E}(a, v, \varepsilon), a]>0\} .\right.
$$

This notion generalizes the classical tangent cone

$$
\operatorname{Tan}(S, a)=\bigcap_{\varepsilon>0}\{v: a \in \operatorname{Clos}[S \cap \mathbf{E}(a, v, \varepsilon)]\}
$$

of a subset $S$ of $\mathbf{R}^{n}$ at $a$, because

$$
\operatorname{Tan}(S, a)=\operatorname{Tan}^{0}\left(\mathcal{H}^{0} L S, a\right) .
$$

It was one of the basic tasks of geometric measure theory to develop applicable criteria characterizing those measures $\gamma$ for which the cones $\operatorname{Tan}^{m}(\gamma, a)$ associated with $\gamma$ almost all points $a$ are $m$ dimensional vectorspaces. We will soon describe such criteria in terms of rectifiability and projection properties.

In case $S \subset \mathbf{R}^{n}$ and $\gamma$ is a measure over $\mathbf{R}^{n}$ we say that:

$S$ is $m$ rectifiable if and only if either $m=0$ and $S$ is finite or $m$ is a positive integer and there exists a Lipschitzian map of some bounded subset of $\mathbf{R}^{m}$ onto $S$.

$S$ is $(\gamma, m)$ rectifiable if and only if $\gamma(S)<\infty$ and $\gamma$ almost all of $S$ can be covered by some countable family of $m$ rectifiable sets.

$S$ is purely $(\gamma, m)$ unrectifiable if and only if $S$ contains no $m$ rectifiable set $T$ with $\gamma(T)>0$.

Rectifiability generalizes a classical approach to first order smoothness, because [23, §3.2.29] a subset $S$ of $\mathbf{R}^{n}$ with $\mathcal{H}^{m}(S)<\infty$ is $\left(\mathcal{H}^{m}, m\right)$ rectifiable if and only if $\mathcal{H}^{m}$ almost all of $S$ can be covered by some countable family of $m$ dimensional submanifolds of (differentiability) class 1 of $\mathbf{R}^{n}$.

The first nontrivial purely $\left(\mathcal{H}^{1}, 1\right)$ unrectifiable subset of $\mathbf{R}^{2}$ was discovered by Gross. It was a Cartesian product

$$
K \times K \text { with } 0<\mathcal{H}^{1}(K \times K)<\infty,
$$

where $K$ was a compact subset of $\mathbf{R}$ obtained through a modified Cantor construction, removing from each compact interval the central open subinterval half as long. 
We let

$$
\mathbf{O}(n, m)
$$

be the space of all orthogonal injections (inner product preserving linear maps) of $\mathbf{R}^{m}$ into $\mathbf{R}^{n}$. Their adjoints form the space

$$
\mathbf{O}^{*}(n, m)=\left\{h^{*}: h \in \mathbf{O}(n, m)\right\}
$$

of all orthogonal projections of $\mathbf{R}^{n}$ onto $\mathbf{R}^{m}$. The orthogonal group $\mathbf{O}(n)=$ $\mathbf{O}(n, n)$ acts transitively on $\mathbf{O}^{*}(n, m)$ through right multiplication. This action induces a unique invariant measure $\theta_{n, m}^{*}$ over $\mathbf{O}^{*}(n, m)$ with

$$
\boldsymbol{\theta}_{n, m}^{*}\left[\mathbf{O}^{*}(n, m)\right]=1 \text {. }
$$

We define the $m$ dimensional integralgeometric measure [23, \$2.10.15]

$$
g_{1}^{m}
$$

over $\mathbf{R}^{n}$ as the Borel regular measure such that

$$
g_{1}^{m}(B)=\int_{\mathbf{O}^{*}(n, m)} \int_{\mathbf{R}^{m}} \mathcal{H}^{0}\left(B \cap p^{-1}\{y\}\right) d \mathscr{L}^{m} y d \theta_{n, m}^{*} p / \boldsymbol{\beta}_{1}(n, m)
$$

for every Borel subset $B$ of $\mathbf{R}^{n}$, where

$$
\boldsymbol{\beta}_{1}(n, m)=\frac{\Gamma[(m+1) / 2] \cdot \Gamma[(n-m+1) / 2]}{\Gamma[(n+1) / 2] \cdot \Gamma[1 / 2]} .
$$

In this formula for $g_{1}^{m}(B)$ we integrate the varying number of points of intersection of $B$ with arbitrary $n-m$ dimensional affine subspaces $p^{-1}\{y\}$ of $\mathbf{R}^{n}$, generalizing Crofton's classical method for finding the length of a plane curve, and also Cauchy's method for finding the area of the boundary of a convex solid.

All the concepts which we have introduced are joined in the following structure theorem $[23,83.3]$.

If $\gamma$ is a Radon measure over $\mathbf{R}^{n}$,

$$
\begin{array}{r}
\gamma\left(\mathbf{R}^{n}\right)<\infty, m \text { is a positive integer, } \\
0<\Theta^{* m}(\gamma, a)<\infty \text { for } \gamma \text { almost all } a, \\
E=\left\{a: \operatorname{Tan}^{m}(\gamma, a) \cap Y \subset\{0\}\right. \text { for some } \\
\left.n-m \text { dimensional vectorsubspace } Y \text { of } \mathbf{R}^{n}\right\},
\end{array}
$$

then:

(1) $E$ is a Borel set and $(\gamma, m)$ rectifiable.

(2) For $\gamma$ almost all $a$ in $E, \operatorname{Tan}^{m}(\gamma, a)$ is an $m$ dimensional vectorsubspace of $\mathbf{R}^{n}$ and $\Theta^{* m}(\gamma, a)=\Theta_{*}^{m}(\gamma, a)$.

(3) Whenever $S \subset E, \gamma(S)>0$ implies $\mathcal{L}^{m}[p(S)]>0$ for $\theta_{n, m}^{*}$ almost all $p$, hence $\mathbb{G}_{1}^{m}(S)>0$.

(4) $\mathbf{R}^{n} \sim E$ is purely $(\gamma, m)$ unrectifiable.

(5) $\mathcal{L}^{m}\left[p\left(\mathbf{R}^{n} \sim E\right)\right]=0$ for $\boldsymbol{\theta}_{n, m}^{*}$ almost all $p$, hence

$$
g_{1}^{m}\left(\mathbf{R}^{n} \sim E\right)=0 .
$$


This result was proved first for $m=1$ and $n=2$ by Besicovitch, A. P. Morse and Randolph, then for arbitrary dimensions by Federer. It has the corollary that the following three conditions are equivalent:

(I) For $\gamma$ almost all $a$,

$\operatorname{Tan}^{m}(\gamma, a)$ is an $m$ dimensional vectorspace.

(II) $\mathbf{R}^{n}$ is $(\gamma, m)$ rectifiable.

(III) Whenever $S \subset \mathbf{R}^{n}, \Phi_{1}^{m}(S)=0$ implies $\gamma(S)=0$.

When $\gamma=\mathfrak{H}^{m}\left\llcorner W\right.$ with $\mathcal{H}^{m}(W)<\infty$, the structure theorem implies

$W \cap E$ is $\left(\mathcal{H}^{m}, m\right)$ rectifiable,

$W \sim E$ is purely $\left(\mathcal{H}^{m}, m\right)$ unrectifiable.

It is also known $[23, \S \S 3.2 .19,3.2 .26]$ that

$$
\begin{aligned}
& \Theta^{m}\left(\mathcal{H}^{m}\llcorner W, a)=1 \text { for } \mathcal{H}^{m} \text { almost all a in } W \cap E,\right. \\
& \mathcal{H}^{m}(S)=\mathscr{G}_{1}^{m}(S) \text { whenever } S \subset W \cap E .
\end{aligned}
$$

Combining the last assertion with (5) we infer

$$
\begin{aligned}
& \mathcal{H}^{m}(W)=\mathscr{I}_{1}^{m}(W \cap E)+\mathcal{H}^{m}(W \sim E)=\mathscr{I}_{1}^{m}(W)+\mathcal{H}^{m}(W \sim E), \\
& \mathcal{H}^{m}(W)=\mathscr{F}_{1}^{m}(W) \text { if and only if } W \text { is }\left(\mathcal{H}^{m}, m\right) \text { rectifiable. }
\end{aligned}
$$

More recently it has been shown by Marstrand and Mattila [56] that

$$
\Theta_{*}^{m}\left(\mathcal{H}^{m}\llcorner W, a)<1 \text { for } \mathcal{H}^{m} \text { almost all a in } W \sim E .\right.
$$

The stronger conjecture that $\Theta_{*}^{m}\left(\mathcal{F}^{m} L W, a\right)<\Theta^{* m}\left(\mathcal{H}^{m} L W, a\right)$ for $\mathcal{H}^{m}$ almost all $a$ in $W \sim E$ is still unsettled in case $m>1$; it was verified in case $m=1$ by Besicovitch, A. P. Morse, Randolph and E. F. Moore.

To illustrate quite simply why the theory really needs approximate tangents, rather than ordinary tangents, we consider a countable family $\Omega$ of circles contained in $\mathbf{R}^{2}$ such that

$$
\sum_{C \in \Omega} \mathcal{H}^{1}(C)<\infty \text { and } \bigcup \Omega \text { is dense in } \mathbf{R}^{2} .
$$

Then $\cup \Omega$ is $\left(\mathcal{H}^{1}, 1\right)$ rectifiable and

$$
\operatorname{Tan}(\bigcup \Omega, a)=\mathbf{R}^{2} \quad \text { for } a \in \mathbf{R}^{2},
$$

but, for each $C \in \Omega$,

$$
\operatorname{Tan}^{1}\left(\mathcal{H}^{1}\llcorner\cup \Omega, a)=\operatorname{Tan}(C, a) \text { for } \mathcal{H}^{1} \text { almost all } a \text { in } C .\right.
$$

During his pioneering and penetrating study of purely $\left(\mathcal{H}^{1}, 1\right)$ unrectifiable subsets of the plane, Besicovitch constructed many beautiful examples, of which the following is particularly illuminating: Let $G_{3}, G_{4}, G_{5}, \ldots$ be finite disjointed families of closed circular disks such that $G_{3}$ consists of a single disc with diameter 2 and $G_{k}$ is obtained from $G_{k-1}$ through replacement of each disc $D$ in $G_{k-1}$ by $k$ disjoint subdiscs of $D$ with diameters equal to 
$\operatorname{diam}(D) / k$ and meeting the boundary of $D$ at $k$ equally spaced points. Then the compact set

$$
\begin{gathered}
\Pi=\bigcap_{k=3}^{\infty} \cup G_{k} \text { is purely }\left(\mathcal{H}^{1}, 1\right) \text { unrectifiable, } \\
\mathcal{H}^{1}(\Pi)=2, \quad \mathcal{L}^{1}[p(\Pi)]=0 \text { whenever } p \in \mathbf{O}^{*}(2,1), \\
\operatorname{Tan}^{1}\left(\mathcal { H } ^ { 1 } \llcorner \Pi , a ) = \mathbf { R } ^ { 2 } \text { and } \Theta ^ { * 1 } \left(\mathcal{H}^{1}\llcorner\Pi, a)=1 / 2\right.\right.
\end{gathered}
$$

for $\mathcal{H}^{1}$ almost all $a$ in $\Pi$.

It is not known whether every set with finite $g_{1}^{m}$ measure is $\left(\Phi_{1}^{m}, m\right)$ rectifiable. This problem is equivalent to the question: Does there exist a set $W$ such that

$$
{\vartheta_{1}^{m}}^{m}(W)>0 \text {, but } \Theta^{m}\left(\oiint_{1}^{m}\llcorner W, a)=0 \text { for } a \in \mathbf{R}^{n}\right. \text { ? }
$$

3. Mapping formulas. We now turn to the discussion of integral formulas relating the Hausdorff measures of the domains, images and level sets of Lipschitzian maps. For this purpose, and for later use with differential forms and currents, we first briefly review some Grassmann algebra.

Each vectorspace $V$ (over $\mathbf{R}$ ) is naturally embedded in its exterior algebra

$$
\wedge_{*} V=\bigoplus_{k=0}^{\infty} \wedge_{k} V
$$

the free graded anticommutative algebra with identity generated by $V$, which is characterized (up to isomorphism) by the requirements that $\bigwedge_{0} V=\mathbf{R}, 1$ is the identity of $\wedge_{*} V, \bigwedge_{1} V=V$ and, for every graded anticommutative algebra

$$
A=\bigoplus_{k=0}^{\infty} A_{k}
$$

with identity, every linear map of $V$ into $A_{1}$ extends to a unique identity preserving graded algebra homomorphism of $\bigwedge_{*} V$ into $A$. In particular, every linear map $L: V \rightarrow V^{\prime}$ extends to the induced homomorphism

$$
\wedge_{*} L: \wedge_{*} V \rightarrow \bigwedge_{*} V^{\prime}
$$

whose direct summands are the linear maps

$$
\bigwedge_{k} L: \bigwedge_{k} V \rightarrow \bigwedge_{k} V^{\prime} .
$$

Multiplication in $\wedge_{*} V$ is denoted by the wedge symbol $\wedge$.

One makes $\bigwedge_{*} V$ a Hopf algebra by means of the diagonal homomorphism into $\bigwedge_{*} V \otimes \bigwedge_{*} V$ which maps $v \in V$ onto $v \otimes 1+1 \otimes v$.

The dual algebra is isomorphic to the alternating algebra of $V$,

$$
\wedge^{*} V=\bigoplus_{k=0}^{\infty} \wedge^{k} V
$$

whose summand $\bigwedge^{k} V \simeq \operatorname{Hom}\left(\bigwedge_{k} V, \mathbf{R}\right)$ consists of all real valued alternating $k$ linear functions on $V^{k}$. The product

$$
\phi \wedge \psi \in \wedge^{p+q} V \text { of } \phi \in \wedge^{p} V \text { and } \psi \in \wedge^{q} V
$$


can be computed by the shuffle formula

$$
\begin{gathered}
(\phi \wedge \psi)\left[v_{1}, \ldots, v_{p+q}\right] \\
=\sum_{\sigma \in \operatorname{Sh}(p, q)} \operatorname{index}(\sigma) \cdot \phi\left[v_{\sigma(1)}, \ldots, v_{\sigma(p)}\right] \cdot \psi\left[v_{\sigma(p+1)}, \ldots, v_{\sigma(p+q)}\right]
\end{gathered}
$$

for $v_{1}, \ldots, v_{p+q} \in V$, where $\operatorname{Sh}(p, q)$ consists of those permutations of $\{1, \ldots, p+q\}$ which are increasing on $\{1, \ldots, p\}$ and on $\{p+1, \ldots, p$ $+q\}$.

Every linear map $L: V \rightarrow V^{\prime}$ induces a homomorphism

$$
\wedge^{*} L: \wedge^{*} V^{\prime} \rightarrow \wedge^{*} V
$$

whose summand $\wedge^{k} L: \wedge^{k} V^{\prime} \rightarrow \wedge^{k} V$, dual to $\wedge_{k} L$, is defined by the formula

$$
\left[\wedge^{k} L(\phi)\right]\left[v_{1}, \ldots, v_{k}\right]=\phi\left[L\left(v_{1}\right), \ldots, L\left(v_{k}\right)\right]
$$

for $\phi \in \bigwedge^{k} V^{\prime}$ and $v_{1}, \ldots, v_{k} \in V$.

The bilinear pairing $\langle$,$\rangle of \wedge_{k} V$ and $\wedge^{k} V$ is such that

$$
\left\langle v_{1} \wedge \cdots \wedge v_{k}, \phi\right\rangle=\phi\left(v_{1}, \ldots, v_{k}\right)
$$

whenever $v_{1}, \ldots, v_{k} \in V$ and $\phi \in \wedge^{k} V$.

With $\xi \in \wedge_{p} V$ and $\phi \in \wedge^{q} V$ one associates the interior products

$\xi \downarrow \phi \in \wedge^{q-p} V$ in case $p<q$ so that

$$
\begin{aligned}
& \langle\eta, \xi\lrcorner \phi\rangle=\langle\eta \wedge \xi, \phi\rangle \text { for } \eta \in \wedge_{q-p} V ; \\
& \xi\left\llcorner\phi \in \wedge_{p-q} V \text { in case } p>q\right. \text { so that } \\
& \left\langle\xi\llcorner\phi, \psi\rangle=\langle\xi, \phi \wedge \psi\rangle \text { for } \psi \in \wedge^{p-q} V .\right.
\end{aligned}
$$

One of ten refers to elements of $\wedge_{k} V$ as $k$ vectors of $V$, and to elements of $\wedge^{k} V$ as $k$ covectors of $V$.

A $k$ vector $\xi$ representable as an exterior product

$$
\xi=v_{1} \wedge \cdots \wedge v_{k} \text { with } v_{1}, \ldots, v_{k} \in V
$$

is call simple; the associated vectorspace

$$
\{x: x \in V \text { and } x \wedge \xi=0\}
$$

has $v_{1}, \ldots, v_{k}$ as base vectors provided $\xi \neq 0$.

To each norm || on $V$ correspond dual norms on $\wedge_{k} V$ and $\wedge^{k} V$ defined as follows:

The mass $\|\xi\|$ of a $k$ vector $\xi$ is the infimum of the set of numbers

$$
\sum_{i=1}^{N}\left|v_{i, 1}\right| \cdot\left|v_{i, 2}\right| \cdot \ldots \cdot\left|v_{i, k}\right|
$$

corresponding to all finite sum representations

$$
\xi=\sum_{i=1}^{N} v_{i, 1} \wedge v_{i, 2} \wedge \ldots \wedge v_{i, k}
$$

with $v_{i, j} \in V$ for $i=1, \ldots, N$ and $j=1, \ldots, k$. 
The comass $\|\phi\|$ of a $k$ covector $\phi$ is

$$
\sup \left\{\phi\left(v_{1}, \ldots, v_{k}\right): v_{j} \in V \text { and }\left|v_{j}\right|<1 \text { for } j=1, \ldots, k\right\} .
$$

Whenever $L: V \rightarrow V^{\prime}$ is a linear map of normed vector spaces the dual maps $\bigwedge_{k} L$ and $\wedge^{k} L$ have norms $\left\|\bigwedge_{k} L\right\|=\left\|\wedge^{k} L\right\|$; in case $V$ and $V^{\prime}$ are inner product spaces,

$$
\begin{aligned}
& \left\|\wedge_{\operatorname{dim} V} L\right\|=\left|\operatorname{det}\left(L^{*} \circ L\right)\right|^{1 / 2} \quad \text { if } \operatorname{dim} V<\infty, \\
& \left\|\wedge_{\operatorname{dim} V^{\prime}} L\right\|=\left|\operatorname{det}\left(L \circ L^{*}\right)\right|^{1 / 2} \quad \text { if } \operatorname{dim} V^{\prime}<\infty .
\end{aligned}
$$

For any function $f$ which maps a neighborhood of the point $a$ in $\mathbf{R}^{m}$ into $\mathbf{R}^{v}$ and which is differentiable at $a$, we define the $k$ dimensional Jacobian

$$
J_{k} f(a)=\left\|\wedge_{k} D f(a)\right\| \quad \text { whenever } 0<k \in \mathbf{Z} .
$$

The classical Jacobian is $J_{m} f(a)$, and $\|D f(a)\|=J_{1} f(a)$. We shall see that the Jacobians of all dimensions between 0 and $m$ occur in integral formulas of geometric interest.

Early work in geometric measure theory confirmed the validity of the area formula

$$
\int_{W^{m}} J_{m} f(x) d \mathscr{L}^{m} x=\int_{\mathbf{R}^{p}} \mathcal{H}^{\mathcal{O}}\left(W \cap f^{-1}\{y\}\right) d \mathcal{H}^{m} y
$$

for every Lipschitzian map $f$ of $\mathbf{R}^{m}$ into $\mathbf{R}^{\nu}$ and every $\mathfrak{L}^{m}$ measurable set $W$. In case $f \mid W$ is injective the right integral equals $\mathcal{H}^{m}[f(W)]$. Accordingly the $m$ dimensional Hausdorff measure of an $m$ rectifiable set equals its $m$ dimensional volume in the sense of classical differential geometry.

It is useful to extend the area formula from subsets of $\mathbf{R}^{m}$ to $\left(\mathrm{F}^{m}, m\right)$ rectifiable subsets of $\mathbf{R}^{n}$. For this purpose one employs the following notion of approximate differentiability:

A function $f$, which maps a subset of $\mathbf{R}^{n}$ into $\mathbf{R}^{p}$, is $(\gamma, m)$ approximately differentiable at the point $a$ if and only if there exists a function $g$, which maps a neighborhood of $a$ in $\mathbf{R}^{n}$ into $\mathbf{R}^{\nu}$, such that

$$
\Theta^{m}[\gamma\llcorner\{x: f(x) \neq g(x)\}, a]=0
$$

and $g$ is differentiable at $a$; under these conditions

$$
D g(a) \mid \operatorname{Tan}^{m}(\gamma, a)
$$

is uniquely determined by $\gamma, m, f, a$ and is called the $(\gamma, m)$ approximate differential of $f$ at $a$, denoted

$$
(\gamma, m) \operatorname{ap} D f(a) \text {. }
$$

In case $\operatorname{Tan}^{m}(\gamma, a)$ is a vectorspace we also define the $(\gamma, m)$ approximate Jacobian

$$
(\gamma, m) \operatorname{ap} J_{k} f(a)=\left\|\wedge_{k}(\gamma, m) \operatorname{ap} D f(a)\right\| \quad \text { for } 0<k \in \mathbf{Z} .
$$

We will omit the prefix $(\gamma, m)$ when it is clear from context.

Applying this definition with $\gamma=\mathcal{H}^{m} L W$, one can prove [23, \$§3.2.19, 3.2.20] the general transformation theorem:

If $W$ is an $\left(\mathcal{H}^{m}, m\right)$ rectifiable $\mathcal{H}^{m}$ measurable subset of $\mathbf{R}^{n}$ and $f$ is a 
Lipschitzian map of $W$ into $\mathbf{R}^{\nu}$, then $f$ is $\left(\mathcal{H}^{m}\llcorner W, m)\right.$ approximately differentiable at $\mathcal{F H}^{m}$ almost all points of $W$ and

$$
\int_{W} \psi[f(x)] \cdot \operatorname{ap} J_{m} f(x) d \mathcal{H}^{m} x=\int_{\mathbf{R}^{\nu}} \psi(y) \cdot \mathcal{H}^{0}\left(W \cap f^{-1}\{y\}\right) d \mathcal{H}^{m} y
$$

for every real valued function $\psi$ on $\mathbf{R}^{\boldsymbol{\nu}}$; moreover

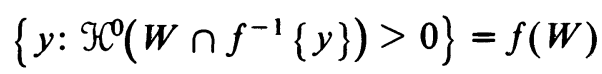

is an $\left(\mathcal{H}^{m}, m\right)$ rectifiable subset of $\mathbf{R}^{p}$, and $W \cap f^{-1}\{y\}$ is finite for $\mathcal{H}^{m}$ almost all $y$ in $\mathbf{R}^{\nu}$.

In case $f(W)$ has Hausdorff dimension less than $m$, the area formula gives very little information. It is rather surprising that quite basic facts about the measure theoretic behavior of dimension lowering maps were not discovered before 1957, when I proved the coarea formula

$$
\int_{W} J_{\mu} f(x) d \mathcal{L}^{m} x=\int_{\mathbf{R}^{\mu}} \mathcal{F}^{\mathcal{F}^{m-\mu}}\left(W \cap \mathcal{f}^{-1}\{y\}\right) d \mathcal{L}^{\mu} y
$$

for every Lipschitzian map $f$ of $\mathbf{R}^{m}$ into $\mathbf{R}^{\mu}$, with $m \geqslant \mu$, and every $\mathfrak{L}^{m}$ measurable set $W$. Area and coarea may be considered dual because they usefully apply to maps of an $m$ dimensional space into spaces with dimensions at least $m$ or at most $m$, respectively. Also, at a crucial point in my initial work on coarea I treated the null set of $J_{\mu} f$ by consciously dualizing Kolmogoroff's treatment of the null set of $J_{m} f$ in his work on area.

Further studies [23, \$\$3.2.22, 3.2.31, 3.2.32] of coarea in more general situations produced the following result:

If $W$ is an $\left(\mathcal{H}^{m}, m\right)$ rectifiable $\mathcal{H}^{m}$ measurable subset of $\mathbf{R}^{n}, f$ is a Lipschitzian map of $W$ into $\mathbf{R}^{\nu}, \mu \in \mathbf{Z}, 0 \leqslant \mu \leqslant m$, and $S$ is any Borel subset of $\mathbf{R}^{\nu}$ with $\mathcal{H}^{\mu}(S)<\infty$, then

$$
\mathcal{H}^{m}\left[W \cap f^{-1}(S) \cap\{x: \operatorname{dim}[\operatorname{im} \text { ap } D f(x)]>\mu\}\right]=0
$$

and

$$
\int_{W \cap f^{-1}(S)} \phi(x) \cdot \operatorname{ap} J_{\mu} f(x) d \mathcal{H}^{m} x=\int_{S} \int_{W \cap f^{-1}\{y\}} \phi(x) d \mathcal{H}^{m-\mu} x d \mathcal{H}^{\mu} y
$$

for every real valued $\mathcal{H C}^{m} L W$ integrable function $\phi$ on $W$; moreover

$$
\left\{y: \mathcal{H}^{m-\mu}\left(W \cap f^{-1}\{y\}\right)>\lambda\right\} \text { is }\left(\mathcal{F C}^{\mu}, \mu\right) \text { rectifiable }
$$

for every positive number $\lambda$, and $W \cap f^{-1}\{y\}$ is $\left(\mathcal{H}^{m-\mu}, m-\mu\right)$ rectifiable and $\mathcal{H}^{m-\mu}$ measurable for $\mathcal{H}^{\mu}$ almost all $y$ in $\mathbf{R}^{\nu}$.

We observe that since $\mathcal{F}^{\mu}(S)<\infty$ there exists an $\left(\mathcal{F}^{\mu}, \mu\right)$ rectifiable Borel subset $Z$ of $S$ such that $S \sim Z$ is purely $\left(\mathcal{H}^{\mu}, \mu\right)$ unrectifiable, and that $S \sim Z$ contributes to neither integral because

$$
\mathcal{F}^{\mu}\left[(S \sim Z) \cap\left\{y: \mathcal{F}^{(m-\mu}\left(W \cap f^{-1}\{y\}\right)>0\right\}\right]=0
$$

while [23, \$3.2.21] implies

$$
\mathcal{H}^{m}\left[W \cap f^{-1}(S \sim Z) \cap\left\{x: \operatorname{ap} J_{\mu} f(x)>0\right\}\right]=0 .
$$


Of course, the integral formula remains valid for any countable union $S$ of Borel subsets of $\mathbf{R}^{\nu}$ with finite $\mathcal{H}^{\mu}$ measures. In case $\mu=m$ the formula holds for every Borel subset $S$ of $\mathbf{R}^{\nu}$. However, in case $\mu<m$ it can happen that

$$
\int_{W \cap f^{-1}(S)} J_{\mu} f d \mathcal{H}^{m}>0=\int_{S} \mathcal{H}^{m-\mu}\left(W \cap f^{-1}\{y\}\right) d \mathcal{F}^{\mu} y
$$

even when $f$ is infinitely differentiable and $S$ is a compact set with Hausdorff dimension $\mu$. (For example take $S=f(T \sim V)$ in [23, 83.2.33].) When $W$ and $f$ are analytic the integral formula holds for every Borel subset $S$ of $\mathbf{R}^{\nu}$.

We now understand that the essential hypotheses for the transformation of integrals under a differentiable map $f$ include some finiteness condition on the measure of the subset $S$ of the target space, which in turn implies a bound on the rank of the differential of $f$ on the inverse image $f^{-1}(S)$.

The opposite problem is to estimate the measure of the direct image of a set on which the rank of the differential of $f$ has a given bound. Whitney, A. P. Morse, Sard and Federer [23, \$\$3.4.3, 3.4.4] found the following solution:

If $j \geqslant 1$ and $m>\mu \geqslant 0$ are integers, $f$ is a function of (differentiability) class $j$ mapping an open subset of $\mathbf{R}^{m}$ into any normed vectorspace, and

$$
B=\{x: \operatorname{dim}[\operatorname{im} D f(x)] \leqslant \mu\},
$$

then

$$
\mathcal{F}^{\mu+(m-\mu) / j}[f(B)]=0,
$$

but for $0 \leqslant s<\mu+(m-\mu) / j$ it can happen that $\mathcal{F}^{s}[f(B)]=\infty$.

4. Gauss-Green formula. Certain subsets $A$ of $\mathbf{R}^{n}$ have the property that, for every smooth vectorfield $\xi$ on $\mathbf{R}^{n}$ with compact support,

$$
\int_{A} \operatorname{div} \xi(x) d \mathscr{L}^{n} x
$$

can be computed by integration of $\xi$ over the boundary of $A$. This idea has several precise and useful formulations. Analysts think of a linear operator. Geometers think of exterior normals, or of approximating $A$ by elementary solids. Thirty years ago the various approaches seemed disparate, but now we know that they are all equivalent.

Much of the theory hinges on the following concept: An exterior normal of $A$ at the point $b$ is defined as a vector

$$
u \in \mathbf{S}^{n-1}=\mathbf{R}^{n} \cap\{w:|w|=1\}
$$

which satisfies the two equations

$$
\begin{aligned}
& \Theta^{n}\left[\mathfrak{L}^{n} L\{x:(x-b) \bullet u>0\} \cap A, b\right]=0, \\
& \Theta^{n}\left[\mathfrak{L}^{n} L\{x:(x-b) \bullet u<0\} \sim A, b\right]=0 ;
\end{aligned}
$$

in case $A$ is $\mathfrak{L}^{n}$ measurable these equations hold if and only if

$$
\operatorname{Tan}^{n}\left(\complement^{n} L A, b\right)=\mathbf{R}^{n} \cap\{v: v \bullet u \leqslant 0\} .
$$

There exists at most one exterior normal of $A$ at $b$. We denote 


$$
\begin{aligned}
& \mathbf{n}(A, b)=u \text { if } A \text { has the exterior normal } u \text { at } b, \\
& \mathbf{n}(A, b)=0 \text { if } A \text { has no exterior normal at } b .
\end{aligned}
$$

It is significant that my notion of exterior normal involves only the measuretheoretic behavior of $A$ with respect to $\varrho^{n}$, and imposes no a priori topological restrictions on $A$. The boundary of $A$ is not even mentioned. This flexible notion permits the Gauss-Green formula to find its own optimal conditions, consistent with geometric intuition and fulfilling natural requirements of functional analysis. The following general theorem [23, \$\$.5.6, 4.5.11] summarizes results obtained by De Giorgi and Federer:

If $A$ is an $\mathfrak{L}^{n}$ measurable subset of $\mathbf{R}^{n}$ and

$$
Z=\left\{x: \Theta^{* n}\left(\mathfrak{L}^{n}\llcorner A, x)>0 \text { and } \Theta^{* n}\left(\mathfrak{L}^{n}\left\llcorner\mathbf{R}^{n} \sim A, x\right)>0\right\},\right.\right.
$$

then the the following five conditions are equivalent:

(1) For every compact $K \subset \mathbf{R}^{n}$ there exists $M \in \mathbf{R}$ such that

$$
\int_{A} \operatorname{div} \xi(x) d \mathfrak{L}^{n} x \leqslant M \sup \{|\xi(x)|: x \in K\}
$$

for every Lipschitzian vectorfield $\xi$ on $\mathbf{R}^{n}$ with spt $\xi \subset K$.

(2) There exist a Radon measure $\gamma$ over $\mathbf{R}^{n}$ and a bounded $\gamma$ measurable covectorfield $\kappa$ such that

$$
\int_{A} \operatorname{div} \xi(x) d \mathfrak{L}^{n} x=\int\langle\xi(x), \kappa(x)\rangle d \gamma x
$$

for every Lipschitzian vectorfield $\xi$ on $\mathbf{R}^{n}$ with compact support.

$$
\int_{A} \operatorname{div} \xi(x) d \mathscr{L}^{n} x=\int \xi(x) \bullet \mathbf{n}(A, x) d \mathcal{H}^{n-1} x
$$

for every Lipschitzian vectorfield $\xi$ on $\mathbf{R}^{n}$ with compact support.

(4) $g_{1}^{n-1}(K \cap Z)<\infty$ for every compact $K \subset \mathbf{R}^{n}$.

(5) There exists a sequence of subsets $A_{j}$ of $\mathbf{R}^{n}$ with polyhedral (or smooth) boundaries such that, for every compact $K \subset \mathbf{R}^{n}$,

$$
\lim _{j \rightarrow \infty} \mathfrak{L}^{n}\left(K \cap\left[\left(A_{j} \sim A\right) \cup\left(A \sim A_{j}\right)\right]\right)=0
$$

and

$$
\limsup _{j \rightarrow \infty} \mathcal{H}^{n-1}\left(K \cap \text { Bdry } A_{j}\right)<\infty .
$$

Moreover, in case these conditions hold, then

$$
K \cap Z \text { is }\left(\mathcal{H}^{n-1}, n-1\right) \text { rectifiable }
$$

for every compact $K \subset \mathbf{R}^{n}$, and

$$
\mathbf{n}(A, x) \in \mathbf{S}^{n-1} \text { with } \operatorname{Tan}^{n-1}\left(\mathcal{H}^{n-1}\llcorner Z, x)=\{v: v \bullet \mathbf{n}(A, x)=0\}\right.
$$

for $\mathcal{H}^{n-1}$ almost all $x$ in $Z$.

One should think of $Z$ as the measuretheoretic boundary of $A$, with respect to $\mathfrak{L}^{n}$. Since $Z$ is contained in the topological boundary of $A$, which we denote Bdry $A$, our condition (4) is implied by the hypothesis 


$$
\mathcal{F}^{n-1}(K \cap \operatorname{Bdry} A)<\infty \quad \text { for every compact } K \subset \mathbf{R}^{n} .
$$

However, this hypothesis demands much more than our condition (4). For example, (4) holds in case $A$ is the complement of an arbitrary set with $\mathfrak{L}^{n}$ measure zero. One can even construct [23, §4.2.25] a subset $\Omega$ of $\mathbf{R}^{3}$ such that Bdry $\Omega$ is homeomorphic to $\mathbf{S}^{2}$ and

$$
\mathfrak{L}^{3}(\text { Bdry } \Omega)>0,
$$

but (4) holds with $A$ replaced by $\Omega$.

5. Weakly differentiable functions. The differential of a smooth real valued function $f$ on $\mathbf{R}^{n}$ is characterized by the fact that, for every Lipschitzian vectorfield $\xi$ on $\mathbf{R}^{n}$ with compact support,

$$
\int\langle\xi(x), D f(x)\rangle d \mathscr{L}^{n} x=\int-\operatorname{div} \xi(x) \cdot f(x) d \mathscr{L}^{n} x .
$$

Since the right integral remains meaningful even when $f$ is not smooth but only locally $\mathfrak{L}^{n}$ summable, this linear function of $\xi$ may be regarded as a measuretheoretic weak differential of $f$, provided it satisfies the boundedness hypothesis of the Riesz representation theorem.

Weakly differentiable functions have appeared in various guises for fifty years, first in the theory of Lebesgue area and later in partial differential equations. They have been characterized alternately by their total variation along families of parallel lines, by their area bounded smooth approximability, by the $n-1$ dimensional measures of their (extended) level sets, and by the $n$ dimensional measure of their (extended) graph. Despite this long history, some of the most interesting global geometric characterizations and, more remarkably, the precise local analytic properties of such functions were discovered only during the last decade.

The following theorem [23, §4.5] describes weakly differentiable functions in various ways and lists some consequent properties.

If $f$ is a real valued $\mathfrak{L}^{n}$ measurable function such that

$$
\int_{K}|f(x)| d \mathfrak{L}^{n} x<\infty \text { for every compact } K \subset \mathbf{R}^{n},
$$

with the lower and upper $\mathfrak{L}^{n}$ approximate limits

$$
\begin{aligned}
& \lambda(x)=\sup \left\{y: \Theta^{n}\left[\mathfrak{L}^{n} L\{z: f(z)<y\}, x\right]=0\right\}, \\
& \mu(x)=\inf \left\{y: \Theta^{n}\left[\mathcal{L}^{n} L\{z: f(z)>y\}, x\right]=0\right\}
\end{aligned}
$$

at each point $x$ in $\mathbf{R}^{n}$, and if

$$
\begin{aligned}
& A=\left(\mathbf{R}^{n} \times \mathbf{R}\right) \cap\{(x, y): f(x)>y\}, \\
& C=\left(\mathbf{R}^{n} \times \mathbf{R}\right) \cap\{(x, y): \lambda(x) \leqslant y \leqslant \mu(x)\},
\end{aligned}
$$

then the following seven conditions are equivalent:

(1) For every compact $K \subset \mathbf{R}^{n}$ there exists $M \in \mathbf{R}$ such that

$$
\int-\operatorname{div} \xi(x) \cdot f(x) d \mathfrak{L}^{n} x \leqslant M \sup \{|\xi(x)|: x \in K\}
$$


for every Lipschitzian vectorfield $\xi$ on $\mathbf{R}^{n}$ with spt $\xi \subset K$.

(2) There exist a Radon measure $\gamma$ over $\mathbf{R}^{n}$ and a bounded $\gamma$ measurable covectorfield $\kappa$ such that

$$
\int-\operatorname{div} \xi(x) \cdot f(x) d \mathfrak{L}^{n} x=\int\langle\xi(x), \kappa(x)\rangle d \gamma x
$$

for every Lipschitzian vectorfield $\xi$ on $\mathbf{R}^{n}$ with compact support.

(3) $\int-\operatorname{div} \xi(x) \cdot f(x) d \mathscr{L}^{n} x=\int(\xi(x), 0) \bullet \mathbf{n}[A,(x, y)] d \mathcal{H}^{n}(x, y)$

for every Lipschitzian vectorfield $\xi$ on $\mathbf{R}^{n}$ with compact support.

(4) $\mathscr{G}_{1}^{n}\{(x, y):(x, y) \in C$ and $x \in K\}<\infty$ for every compact $K \subset \mathbf{R}^{n}$.

(5) For every compact $K \subset \mathbf{R}^{n}$,

$$
\int g_{1}^{n-1}\{x:(x, y) \in C \text { and } x \in K\} d \mathcal{L}^{1} y<\infty .
$$

(6) There exists a sequence of locally Lipschitzian real valued functions $f_{j}$ on $\mathbf{R}^{n}$ such that, for every compact $K \subset \mathbf{R}^{n}$,

$$
\lim _{j \rightarrow \infty} \int_{K}\left|f_{j}(x)-f(x)\right| d L^{n} x=0
$$

and

$$
\underset{j \rightarrow \infty}{\limsup } \int_{K}\left\|D f_{j}(x)\right\| d \mathscr{L}^{n} x<\infty .
$$

(7) There exists a real valued function $g$ on $\mathbf{R}^{n}$ such that

$$
g(x)=f(x) \text { for } \mathrm{L}^{n} \text { almost all } x
$$

and, for $i \in\{1, \ldots, n\},-\infty<a<b<\infty$ and every compact $H \subset \mathbf{R}^{n-1}$,

$$
\int_{H} \mathbf{V}_{t=a}^{t=b} g\left(w_{1}, \ldots, w_{i-1}, t, w_{i}, \ldots, w_{n-1}\right) d \mathfrak{L}^{n-1} w<\infty
$$

in case $n>1$, while $\mathrm{V}_{a}^{b} g<\infty$ in case $n=1$.

Here $\mathbf{V}$ means total variation.

Moreover, in case these seven equivalent conditions hold, the following consequences may be drawn:

(I) For $\mathcal{F}^{n}$ almost all $(x, y)$ in $\mathbf{R}^{n} \times \mathbf{R}$,

$$
(x, y) \in C \text { if and only if } \mathbf{n}[A,(x, y)] \in \mathbf{S}^{n} .
$$

Thus the extended graph $C$ of $f$ is $\mathcal{F H}^{n}$ almost equal to the measuretheoretic boundary of $A$ with respect to $\mathfrak{L}^{n} \times \mathcal{L}^{1}$, for the purpose of the Gauss-Green formula.

(II) For every open $U \subset \mathbf{R}^{n}$,

$$
\mathcal{F}^{n}\{(x, y):(x, y) \in C \text { and } x \in U\}
$$

equals the least of the numbers

$$
\liminf _{j \rightarrow \infty} \int_{U}\left(1+\left\|D f_{j}(x)\right\|^{2}\right)^{1 / 2} d \mathscr{L}^{n} x
$$


corresponding to all sequences of functions $f_{j}$ as in (6).

Accordingly the $n$ dimensional measure of the extended graph of $f \mid U$ equals the Lebesgue area of the nonparametric surface corresponding to $f \mid U$.

(III) For every Borel subset $\mathbf{S}$ of $\mathbf{R}^{n}$,

$$
\int \mathscr{G}_{1}^{n-1}\{x:(x, y) \in C \text { and } x \in S\} d \mathcal{L}^{1} y=\int_{S}\|\kappa(x)\| d \gamma x
$$

whenever $\gamma$ and $\kappa$ are as in (2).

In this way the $n-1$ dimensional measures of the extended level sets of $f$ determine the variation of the weak differential of $f$.

$$
\begin{aligned}
\int- & \operatorname{div} \xi(x) \cdot f(x) d \mathscr{L}^{n} x \\
& =\iint \xi(x) \bullet \mathbf{n}[\{z: f(z)<y\}, x] d \mathcal{H}^{n-1} x d \mathcal{L}^{1} y
\end{aligned}
$$

for every Lipschitzian vectorfield $\xi$ on $\mathbf{R}^{n}$ with compact support.

(IV) holds also for some functions which are not weakly differentiable, for example $f(x)=x \sin (1 / x)$. In fact (IV) holds whenever $f$ is locally $\mathfrak{L}^{n}$ summable and the Gauss-Green formula is applicable to $\{x: f(x)<y\}$ for $\mathfrak{L}^{1}$ almost all $y$, because

$$
\begin{aligned}
& \int \operatorname{div} \xi(x) \cdot f^{+}(x) d \mathscr{L}^{n} x=\iint_{0}^{f^{+}(x)} \operatorname{div} \xi(x) d \mathscr{L}^{\mathbf{l} y} d \mathfrak{L}^{n} x \\
& =\int_{\left(\mathbf{R}^{n} \times \mathbf{R}\right) \cap\{(x, y): f(x)>y>0\}} \operatorname{div} \xi(x) d\left(\mathfrak{L}^{n} \times \mathfrak{L}^{1}\right)(x, y) \\
& =\int_{0}^{\infty} \int_{\{x: f(x)>y\}} \operatorname{div} \xi(x) d \mathscr{L}^{n} x d \mathscr{L}^{\mathbf{l} y} \\
& =\int_{0}^{\infty} \int \xi(b) \bullet \mathbf{n}[\{x: f(x)>y\}, b] d \mathcal{H}^{n-1} b d \mathscr{L}^{1} y,
\end{aligned}
$$

and similarly

$$
\begin{aligned}
& \int \operatorname{div} \xi(x) \cdot f^{-}(x) d \mathscr{L}^{n} x \\
& \quad=\int_{-\infty}^{0} \int \xi(b) \cdot \mathbf{n}[\{x: f(x)<y\}, b] d \mathscr{H}^{n-1} b d L^{1} y .
\end{aligned}
$$

The next four properties generalize the classical proposition that a function with locally bounded variation has everywhere finite left and right limits, which differ only on a countable set.

(V) $-\infty<\lambda(x)<\mu(x)<\infty$ for $\mathcal{H}^{n-1}$ almost all $x$ in $\mathbf{R}^{n}$.

(VI) The set $E=\{x: \lambda(x)<\mu(x)\}$ is the union of a countable family of $\left(\mathcal{H}^{n-1}, n-1\right)$ rectifiable sets.

(VII) For $\mathcal{H}^{n-1}$ almost all $x$ in $\mathbf{R}^{n} \sim E$, in case $n>1$, 


$$
\lim _{r \downarrow 0} r^{-n} \int_{\mathbf{B}(x, r)}|f(z)-\lambda(x)|^{n /(n-1)} d \mathscr{L}^{n} z=0 .
$$

(VIII) For $\mathcal{F}^{n-1}$ almost all $x$ in $E$ there exists $u \in \mathbf{S}^{n-1}$ such that, in case $n>1$,

$$
\begin{aligned}
& \lim _{r \downarrow 0} r^{-n} \int_{\mathbf{B}(x, r) \cap N}|f(z)-\lambda(x)|^{n /(n-1)} d \mathcal{L}^{n} z=0, \\
& \lim _{r \downarrow 0} r^{-n} \int_{\mathbf{B}(x, r) \cap P}|f(z)-\mu(x)|^{n /(n-1)} d \mathcal{L}^{n} z=0,
\end{aligned}
$$

where $N=\{z:(z-x) \bullet u<0\}, P=\{z:(z-x) \bullet u>0\}$.

Thus we recognize $\lambda(x)$ and $\mu(x)$ as approximate limits on opposite sides of the plane through $x$ with normal $u$.

Next we discuss an application to Fourier analysis. Whenever $f$ is $\mathfrak{L}^{n}$ summable one defines its Fourier transform $\hat{f}$ by the formula

$$
\hat{f}(\alpha)=\int f(x) \exp (-\mathbf{i} x \bullet \alpha) d \mathscr{L}^{n} x \quad \text { for } \alpha \in \mathbf{R}^{n} .
$$

The Bochner process of spherical summation seeks to represent $f$ in terms of $\hat{f}$ as follows:

Given $\delta>-1$ one uses the factor

$$
K^{\delta}(t)=\left[\sup \left\{1-t^{2}, 0\right\}\right]^{\delta} \text { for } t \in \mathbf{R}
$$

to construct the partial integrals

$$
S_{R}^{\delta}(x)=(2 \pi)^{-n} \int \hat{f}(x) \exp (\mathbf{i} x \bullet \alpha) K^{\delta}(|\alpha| / R) d L^{n} \alpha
$$

for $x \in \mathbf{R}^{n}$ and $R>0$. One tries to prove that, as $R$ approaches $\infty, S_{R}^{\delta}(x)$ converges to $f(x)$, or more generally to a number determined by the limiting behavior of $f$ at $x$.

Bochner generalized Fejér's summability theorem concerning $L^{1}$ summable functions by proving:

If $f$ is $\mathfrak{L}^{n}$ summable and if $\delta>(n-1) / 2$, then

$$
\lim _{R \rightarrow \infty} S_{R}^{\delta}(x)=f(x) \text { for } \mathfrak{L}^{n} \text { almost all } x \text { in } \mathbf{R}^{n} .
$$

Much more recently Federer [24] generalized the Dirichlet-Jordan theorem concerning functions with bounded variation on $\mathbf{R}$ by proving:

If $f$ is $\mathfrak{L}^{n}$ summable and weakly differentiable with finite total variation on $\mathbf{R}^{n}$, which means that $\int\|\kappa\| d \gamma<\infty$ in (2), and if $\delta>(n-3) / 2$, then

$$
\lim _{R \rightarrow \infty} S_{R}^{\delta}(x)=\frac{\lambda(x)+\mu(x)}{2} \text { for } \mathcal{H}^{n-1} \text { almost all } x \text { in } \mathbf{R}^{n} .
$$

Research on weakly differentiable functions has produced far more information than we have time to review here completely. For instance the measure $\gamma L\|\kappa\|$, as in (2), is absolutely continuous with respect to $\mathcal{F C}^{n-1}$ or $\mathfrak{E}^{n}$, respectively, if and only if a function $g$, as in (7), can be chosen 
continuous or absolutely continuous, respectively, along almost all straight lines in $\mathbf{R}^{n}$. However, the extent of our discussion of weakly differentiable functions should suffice to indicate why work on this special topic provided significant motivation and experience for the development of the general theory to which we now turn our attention.

6. Currents. It is a common procedure of contemporary mathematics to probe for the geometric shape and analytic nicety of very general objects by studying their interaction with infinitely smooth test functions. For the theory of $\boldsymbol{m}$ dimensional currents in $\mathbf{R}^{n}$ the test functions are differential forms of degree $m$, which means functions whose values are $m$ covectors of $\mathbf{R}^{n}$. We define

$$
\mathcal{E}^{m}(U)
$$

as the vectorspace of all differential forms of degree $m$ and (differentiability) class $\infty$ on the open subset $U$ of $\mathbf{R}^{n}$, topologized by the seminorms

$$
\nu_{K}^{i}(\phi)=\sup \left\{\left\|D^{j} \phi(x)\right\|: 0 \leqslant j \leqslant i \text { and } x \in K\right\}
$$

corresponding to all nonnegative integers $i$ and all compact subsets $K$ of $U$. Each of the closed subspaces

$$
\mathscr{Q}_{K}^{m}(U)=\mathcal{E}^{m}(U) \cap\{\phi: \operatorname{spt} \phi \subset K\}
$$

is relatively topologized by the seminorms $\nu_{K}^{i} \mid \bigoplus_{K}^{m}(U)$, but their union

$$
\text { श) }^{m}(U)=\mathcal{E}^{m}(U) \cap\{\phi: \text { spt } \phi \text { is a compact subset of } U\}
$$

is endowed with the largest topology making the inclusion maps from all the spaces $\mathscr{Q}_{K}^{m}(U)$ continuous. An $m$ dimensional current in $U$ is, by definition, a continuous real valued linear function on $\mathscr{D}^{m}(U)$. These currents are the elements of the vectorspace

$$
\mathscr{Q}_{m}(U)
$$

endowed with the weak topology generated by the sets

$$
\mathscr{D}_{m}(U) \cap\{S: \rho<S(\phi)<\sigma\}
$$

corresponding to all $\phi \in \mathcal{Q}^{m}(U)$ and $\rho, \sigma \in \mathbf{R}$.

The support of a current $S \in \mathscr{D}_{m}(U)$ is the smallest relatively closed subset $C$ of $U$ such that $S(\phi)=0$ for all $\phi \in \mathscr{Q}^{m}(U)$ with spt $\phi \subset U \sim C$.

An $m$ dimensional geometric surface can be represented analytically by a current, because the surface is determined by the operation of integrating arbitrary smooth differential forms of degree $m$ over the surface. Later we will give a precise definition of the class of rectifiable currents, those currents which represent in our view the surfaces most useful for the calculus of variations. First we must discuss parts of the general theory of currents in order to provide an appropriate logical frame for our geometric constructions $[23,84.1]$.

The interior and alternating multiplications in the Grassman algebra of $\mathbf{R}^{\boldsymbol{n}}$ yield dual operations on currents:

If $S \in \mathscr{D}_{m}(U)$ and $\xi: U \rightarrow \bigwedge_{k} \mathbf{R}^{n}$ is of class $\infty$, then

$$
S \wedge \xi \in \mathscr{D}_{m+k}(U), \quad(S \wedge \xi)(\phi)=S(\xi \dashv \phi) \text { for } \phi \in \mathscr{D}^{m+k}(U) \text {. }
$$


If $S \in \mathscr{D}_{m}(U)$ and $\psi \in \mathcal{E}^{k}(U)$ with $m \geqslant k$, then

$$
S\left\llcorner\psi \in \mathscr{D}_{m-k}(U), \quad\left(S\llcorner\psi)(\phi)=S(\psi \wedge \phi) \quad \text { for } \phi \in \mathscr{Q}^{m-k}(U) .\right.\right.
$$

One uses the first order partial differentiations $D_{1}, \ldots, D_{n}$ inducing continuous endomorphisms of $\mathscr{Q}^{m}(U)$ to define

$$
\begin{aligned}
& D_{i} S \in \mathscr{D}_{m}(U) \quad \text { whenever } S \in \mathscr{D}_{m}(U), \\
& D_{i} S(\phi)=S\left(-D_{i} \phi\right) \text { for } \phi \in \mathscr{D}^{m}(U) .
\end{aligned}
$$

Among the simplest currents are the following:

To each $a \in U$ corresponds the point mass

$\boldsymbol{\delta}_{a} \quad$ (also denoted [a] $) \in \mathscr{D}_{0}(U), \quad \boldsymbol{\delta}_{a}(\phi)=\phi(a)$ for $\phi \in \mathscr{D}^{0}(U)$.

Whenever $\{(1-t) a+t b: 0 \leqslant t \leqslant 1\} \subset U$ we define the oriented line segment

$$
\begin{gathered}
{[a, b] \in \mathscr{D}_{1}(U),} \\
{[a, b](\phi)=\int_{0}^{1}\langle b-a, \phi[(1-t) a+t b]\rangle d \mathcal{L}^{1} t \text { for } \phi \in \mathscr{D}^{1}(U) .}
\end{gathered}
$$

To every Radon measure $\gamma$ over $U$ corresponds a 0 dimensional current denoted by the same symbol, so that

$$
\gamma(\phi)=\int \phi d \gamma \quad \text { for } \phi \in \mathscr{Q}^{0}(U) .
$$

Using the standard base vectors $e_{1}, \ldots, e_{n}$ of $\mathbf{R}^{n}$ to define the $n$ vectorfield $\xi$ orienting $\mathbf{R}^{n}$,

$$
\xi(x)=e_{1} \wedge \cdots \wedge e_{n} \in \wedge_{n} \mathbf{R}^{n} \text { for } x \in \mathbf{R}^{n},
$$

we construct the Euclidean current $\mathbf{E}^{n}=\mathfrak{L}^{n} \wedge \xi \in \mathscr{D}_{n}\left(\mathbf{R}^{n}\right)$,

$$
\mathbf{E}^{n}(\phi)=\int\left\langle e_{1} \wedge \cdots \wedge e_{n}, \phi(x)\right\rangle d \mathfrak{L}^{n} x \text { for } \phi \in \mathscr{Q}^{n}\left(\mathbf{R}^{n}\right) .
$$

Next we review four constructions basic for geometry, namely the boundary operator, homomorphisms induced by smooth proper maps, Cartesian products and joins of currents.

For $\phi \in \mathcal{E}^{m}(U)$ the exterior derivative $d \phi \in \mathcal{E}^{m+1}(U)$ is given by the formula

$$
\left\langle v_{1} \wedge \cdots \wedge v_{m+1}, d \phi(x)\right\rangle=\sum_{i=1}^{m+1}\left\langle v^{(i)},\left\langle v_{i}, D \phi(x)\right\rangle\right\rangle
$$

with

$$
v^{(i)}=(-1)^{i-1} v_{1} \wedge \cdots \wedge v_{i-1} \wedge v_{i+1} \wedge \cdots \wedge v_{m+1}
$$

whenever $x \in U$ and $v_{1}, \ldots, v_{m+1} \in \mathbf{R}^{n}$. Dually the boundary operator maps $T \in \mathscr{D}_{m+1}(U)$ onto $\partial T \in \mathscr{D}_{m}(U)$ so that

$$
\partial T(\phi)=T(d \phi) \quad \text { for } \phi \in \mathscr{Q}^{m}(U) .
$$

Using the standard coordinate functions $X_{1}, \ldots, X_{n}$ on $\mathbf{R}^{n}$ one computes

$$
d \phi=\sum_{i=1}^{n} D X_{i} \wedge D_{i} \phi \quad \text { and } \quad \partial T=-\sum_{i=1}^{n}\left(D_{i} T\right)\left\llcorner D X_{i}\right. \text {. }
$$


Every map $f: U \rightarrow U^{\prime}$ of class $\infty$, where $U$ and $U^{\prime}$ are open subsets of Euclidean spaces, induces a homomorphism

$$
f^{\#}: \mathcal{E}^{m}\left(U^{\prime}\right) \rightarrow \mathcal{E}^{m}(U)
$$

such that $\left[f^{\#} \phi\right](x)=\left[\bigwedge^{m} D f(x)\right] \phi[f(x)]$ for $\phi \in \mathcal{E}^{m}\left(U^{\prime}\right)$ and $x \in U$. In case $S \in \mathscr{D}_{m}(U)$ and $f \mid$ spt $S$ is proper one defines

$$
f_{\#} S \in \mathscr{D}_{m}\left(U^{\prime}\right)
$$

so that $\left[f_{\#} S\right](\phi)=S\left(\alpha \wedge f^{\#} \phi\right)$ whenever $\phi \in \mathscr{Q}^{m}\left(U^{\prime}\right)$ and $\alpha \in \mathscr{D}^{0}(U)$ with spt $S \cap f^{-1}(\operatorname{spt} \phi) \subset \operatorname{Int}\{x: \alpha(x)=1\}$. The linear maps $f^{\#}$ and $f_{\#}$ commute with $d$ and $\partial$, respectively.

In case $f: U \hookrightarrow U^{\prime}$ is an inclusion map of open subsets of $\mathbf{R}^{n}$, then $f_{\#}$ maps

$$
\operatorname{dmn} f_{\#}=\mathscr{D}_{m}(U) \cap\left\{S: \text { spt } S \text { is relatively closed in } U^{\prime}\right\}
$$

homeomorphically onto

$$
\operatorname{im} f_{\#}=\mathscr{D}_{m}\left(U^{\prime}\right) \cap\{T: \text { spt } T \subset U\},
$$

and one often fails to distinguish notationally between $S$ and $f_{\#} S$; this applies in particular to currents with compact support in $U$.

The Cartesian product

$$
S \times T \in \mathscr{D}_{i+j}(U \times V) \text { of } S \in \mathscr{D}_{i}(U) \text { and } T \in \mathscr{D}_{j}(V)
$$

is characterized by the following condition:

If $\phi \in \mathscr{D}^{k}(U)$ and $\psi \in \mathscr{D}^{i+j-k}(V)$, then

$$
\begin{aligned}
& (S \times T)\left(p^{\#} \phi \wedge q^{\#} \psi\right)=S(\phi) T(\psi) \text { in case } k=i, \\
& (S \times T)\left(p^{\#} \phi \wedge q^{\#} \psi\right)=0 \text { in case } k \neq i,
\end{aligned}
$$

where $p(x, y)=x$ and $q(x, y)=y$ for $(x, y) \in U \times V$. One finds that, in case $i+j>0$,

$$
\partial(S \times T)=(\partial S) \times T+(-1)^{i} S \times \partial T
$$

with the first summand omitted if $i=0$, the second if $j=0$. An important consequence is the homotopy formula

$$
h_{1 \#} T-h_{0 \#} T=\partial h_{\#}([0,1] \times T)+h_{\#}([0,1] \times \partial T)
$$

which holds for each infinitely differentiable homotopy

$$
h: \mathbf{R} \times V \rightarrow V^{\prime} \quad \text { with } h_{t}(v)=h(t, v) \text { for }(t, v) \in \mathbf{R} \times V,
$$

provided $h \mid(\{t: 0 \leqslant t \leqslant 1\} \times$ spt $T)$ is proper; if $j=0$ the second summand must be omitted.

The join of two currents $S \in \mathscr{D}_{i}\left(\mathbf{R}^{n}\right)$ and $T \in \mathscr{D}_{j}\left(\mathbf{R}^{n}\right)$ with compact supports is

$$
S * T=F_{\#}(S \times[0,1] \times T) \in \mathscr{D}_{i+1+j}\left(\mathbf{R}^{n}\right)
$$

where $F(x, t, y)=(1-t) x+t y$ for $(x, t, y) \in \mathbf{R}^{n} \times \mathbf{R} \times \mathbf{R}^{n}$. Then

$$
\partial(S * T)=(\partial S) * T-(-1)^{i} S * \partial T
$$

with $(\partial S) * T$ replaced by $S(1) T$ if $i=0, S \star \partial T$ replaced by $T(1) S$ if $j=0$. 
For $a_{0}, a_{1}, \ldots, a_{m} \in \mathbf{R}^{n}$ we inductively define the $m$ dimensional oriented simplex

$$
\left[a_{0}, \ldots, a_{m}\right]=\left[a_{0}\right] *\left[a_{1}, \ldots, a_{m}\right] \in \mathscr{D}_{m}\left(\mathbf{R}^{n}\right)
$$

and compute

$$
\partial\left[a_{0}, \ldots, a_{m}\right]=\sum_{i=0}^{m}(-1)^{i}\left[a_{0}, \ldots, a_{i-1}, a_{i+1}, \ldots, a_{m}\right] .
$$

If $U$ contains the convex hull of $\left\{a_{0}, \ldots, a_{m}\right\}$, then $\left[a_{0}, \ldots, a_{m}\right]$ corresponds to an element of $\mathscr{D}_{m}(U)$, denoted by the same symbol. All such oriented simplexes generate in $\mathscr{D}_{m}(U)$ the additive subgroup

$$
\mathcal{P}_{m}(U)
$$

of $m$ dimensional integral polyhedral chains in $U$, and the vectorsubspace

$$
\mathbf{P}_{m}(U)
$$

of $m$ dimensional (real) polyhedral chains in $U$. if

A current $S \in \mathscr{D}_{m}(U)$ is said to be representable by integration if and only

$$
\|S\|(k)=\sup \left\{S(\phi): \phi \in \mathscr{D}_{m}(U) \text { and }\|\phi\|<k\right\}<\infty
$$

for every nonnegative real valued continuous function $k$ with compact support in $U$. Then $\|S\|$ corresponds to a Radon measure over $U$, by the Riesz representation theorem, and there exists an $\|S\|$ measurable function $\vec{S}$ such that

$$
\begin{gathered}
\vec{S}(x) \in \bigwedge_{m} \mathbf{R}^{n} \quad \text { with }\|\vec{S}(x)\|=1 \text { for }\|S\| \text { almost all } x, \\
S(\phi)=\int\langle\vec{S}(x), \phi(x)\rangle d\|S\| x \text { for } \phi \in \mathscr{D}^{m}(U) .
\end{gathered}
$$

Using the last equation we extend the definitions of $S(\phi), S \wedge \xi, S\llcorner\psi$ to $\|S\|$ summable functions $\phi, \xi, \psi$ and obtain the formula

$$
S=\|S\| \wedge \vec{S}
$$

Accordingly we picture $S$ as composed of a mass distribution and an attached $m$ vectorfield. We write $S\llcorner A=S L \alpha$ when $\alpha$ is the characteristic function of a set $A$. From the theory of derivation of measures it follows for $\|S\|$ almost all $x$ that

$$
\lim _{r \downarrow 0} \frac{[S\llcorner\mathbf{B}(x, r)](\phi)}{\|S\|[\mathbf{B}(x, r)]}=\langle\vec{S}(x), \phi(x)\rangle \text { whenever } \phi \in \mathbb{D}^{m}(U) .
$$

For instance the current $\mathbf{E}^{n}$ is representable by integration,

$$
\left\|\mathbf{E}^{n}\right\|=e^{n} \text { and } \overrightarrow{\mathbf{E}}^{n}(x)=e_{1} \wedge \cdots \wedge e_{n} \text { for } x \in \mathbf{R}^{n} .
$$

To each locally $\mathfrak{L}^{n}$ summable differential form $\psi$ of degree $k<n$ corresponds $\mathbf{E}^{n} L \psi \in \mathscr{D}_{n-k}\left(\mathbf{R}^{n}\right)$; if $\psi$ is Lipschitzian, then

$$
D_{i}\left(\mathbf{E}^{n} L \psi\right)=\mathbf{E}^{n} L D_{i} \psi \text { and } \partial\left(\mathbf{E}^{n} L \psi\right)=(-1)^{n-k-1} \mathbf{E}^{n} L d \psi \text {. }
$$

Therefore many analysts think of currents as generalized differential forms. 
This point of view is very convenient for the theory of partial differential equations. However, for work in geometry it appears preferable to follow the lead of the topologists, who distinguish intuitively as well as logically between cochains and chains. I consciously regard differential forms and currents as dual objects, which are transformed in opposite directions by differentiable maps.

We define the mass of any $S \in \mathscr{D}_{m}(U)$ as

$$
\mathbf{M}(S)=\|S\|(1)=\sup \left\{S(\phi): \phi \in \mathscr{D}^{m}(U) \text { and }\|\phi\|<1\right\},
$$

and infer that the set

$$
\mathbf{M}_{m}(U)=\mathscr{D}_{m}(U) \cap\{S: \text { spt } S \text { is compact and } \mathbf{M}(S)<\infty\}
$$

consists of all those $m$ dimensional currents with compact support which are representable by integration.

We are now ready to begin the discussion of those special classes of currents which have been most useful for geometric measure theory.

We call an $m$ dimensional current $S$ locally normal if and only if $S$ is representable by integration and either $\partial S$ is representable by integration or $m=0$.

We call a current normal if and only if it is locally normal and has compact support. The set

$$
\mathbf{N}_{m}(U)=\mathscr{D}_{m}(U) \cap\{S: S \text { is normal }\}
$$

is the union of the sets

$$
\mathbf{N}_{m, K}(U)=\mathscr{Q}_{m}(U) \cap\{S: S \text { is normal and spt } S \subset K\}
$$

corresponding to all compact $K \subset U$. For $S \in \mathscr{D}_{m}(U)$ we define

$$
\begin{aligned}
& \mathbf{N}(S)=\mathbf{M}(S)+\mathbf{M}(\partial S) \text { in case } m>0, \\
& \mathbf{N}(S)=\mathbf{M}(S) \text { in case } m=0 .
\end{aligned}
$$

For example the $n$ dimensional locally normal currents in $\mathbf{R}^{n}$ are the currents $\mathbf{E}^{n} L \psi$ corresponding to all weakly differentiable real valued functions $\psi$, with

$$
\begin{aligned}
{\left[\partial\left(\mathbf{E}^{n} L \psi\right)\right](\phi) } & =\int\left\langle e_{1} \wedge \cdots \wedge e_{n}, \psi(x) d \phi(x)\right\rangle d \mathcal{L}^{n} x \\
& =(-1)^{n-1} \int \psi(x) \operatorname{div} \xi(x) d \mathcal{L}^{n} x
\end{aligned}
$$

whenever $\phi \in \mathscr{D}^{n-1}\left(\mathbf{R}^{n}\right)$ and $\xi(x)=\left(e_{1} \wedge \cdots \wedge e_{n}\right)\left\llcorner\phi(x)\right.$ for $x \in \mathbf{R}^{n}$.

The current $\mathbf{E}^{n}\left\llcorner A\right.$ corresponding to an $\mathcal{L}^{n}$ measurable set $A$ is locally normal if and only if our version of the Gauss-Green formula holds for $A$.

The vectorspaces $\mathbf{N}_{m, K}(U)$ are complete with respect to the norm $\mathbf{N}$, but hardly ever separable. Often the images of a normal current under two neighboring smooth maps have large $\mathbf{N}$ distance. For these and other reasons it is frequently advantageous to use the (real) flat seminorm

$$
\mathrm{F}_{K}(S)
$$

defined whenever $S \in \mathscr{D}_{m}(U)$ as the supremum of

$$
\left\{S(\phi): \phi \in \mathscr{D}^{m}(U), \nu_{K}^{0}(\phi) \leqslant 1, \nu_{K}^{0}(d \phi)<1\right\} .
$$


In case spt $S \subset K$, then $\mathbf{F}_{K}(S)$ equals the least member (possibly $\infty$ ) of

$$
\left\{\mathbf{M}(S-\partial T)+\mathbf{M}(T): T \in \mathscr{D}_{m+1}(U) \text { with spt } T \subset K\right\} .
$$

The homotopy formula leads to the basic estimate

$$
\mathbf{F}_{K^{\prime}}\left(g_{\#} S-f_{\#} S\right) \leqslant \int|g-f| d\left(c^{m}\|S\|+c^{m-1}\|\partial S\|\right)
$$

for $S \in \mathbf{N}_{m, K}(U)$ and any two infinitely differentiable maps $f$ and $g$ of $U$ into $U^{\prime}$ such that

$$
\begin{gathered}
\{(1-t) f(x)+\operatorname{tg}(x): 0 \leqslant t \leqslant 1, x \in K\} \subset K^{\prime} \subset U^{\prime}, \\
\|D f(x)\| \leqslant c \text { and }\|D g(x)\| \leqslant c \text { for } x \in K .
\end{gathered}
$$

For each compact $K \subset U$ the vectorspace

$$
\mathbf{F}_{m, K}(U)=\text { the } \mathbf{F}_{K} \text { closure of } \mathbf{N}_{m, K}(U) \text { in } \mathscr{D}_{m}(U)
$$

is complete with respect to the flat norm $\mathbf{F}_{K}$. The union of the spaces $\mathbf{F}_{m, K}(U)$ corresponding to all compact $K \subset U$ is the space

$$
\mathbf{F}_{m}(U)
$$

of $m$ dimensional (real) flat chains in $U$. Endowing $\mathbf{F}_{m}(U)$ with the largest topology such that the inclusion maps from all the subspaces $F_{m, K}(U)$ are continuous one obtains the dual space

$$
\mathbf{F}^{m}(U)
$$

of all continuous real valued linear functions on $\mathbf{F}_{m}(U)$, which are called (real) locally flat cochains of degree $m$ of $U$.

The images of flat chains under differentiable maps behave rather well with respect to equi-Lipschitzian convergence [23, §4.1.14]. One can define

$$
f_{\#} S \in \mathbf{F}_{m, K^{\prime}}\left(U^{\prime}\right) \text { for } S \in \mathbf{F}_{m, K}(U)
$$

whenever $f: U \rightarrow U^{\prime}$ is locally Lipschitzian and $K, K^{\prime}$ are compact subsets of $U, U^{\prime}$ with $f(K) \subset$ Int $K^{\prime}$, in such a way that

$$
\lim _{j \rightarrow \infty} \mathbf{F}_{K^{\prime}}\left(f_{\#} S-g_{j \#} S\right)=0
$$

for every sequence of infinitely differentiable maps $g_{j}: U \rightarrow U^{\prime}$ which converge to $f$ uniformly on $K$ and whose first differentials are bounded uniformly on $K$.

This convergence property of flat chains is not shared by arbitrary currents. For instance if

$$
f(x)=0 \text { and } g_{j}(x)=j^{-1} \sin (j x) \text { for } x \in \mathbf{R},
$$

then $f_{\#}\left(\delta_{0} \wedge e_{1}\right)=0$ is not the limit of $g_{j \#}\left(\delta_{0} \wedge e_{1}\right)=\delta_{0} \wedge e_{1}$ with respect to the topology of $\mathscr{D}_{1}(\mathbf{R})$; thus

$$
\delta_{0} \wedge e_{1} \in \mathbf{M}_{1}(\mathbf{R}) \sim \mathbf{F}_{1}(\mathbf{R}) \subset \mathbf{M}_{1}(\mathbf{R}) \sim \mathbf{N}_{1}(\mathbf{R}) .
$$

It follows from the convergence property that

$$
f_{0} \mid \text { spt } S=f_{1} \mid \text { spt } S \text { implies } f_{0 \#} S=f_{1 \#} S
$$

for $S \in \mathbf{F}_{m}(U)$ and locally Lipschitzian maps $f_{0}, f_{1}: U \rightarrow U^{\prime}$.

Flat chains also enjoy very useful measuretheoretic properties: If $S \in$ 
$\mathbf{F}_{m}(U)$, then

$$
g_{1}^{m}(\operatorname{spt} S)=0 \quad \text { implies } S=0 .
$$

If $S \in \mathbf{F}_{m}(U) \cap \mathbf{M}_{m}(U)$ and $E \subset U$, then

$$
g_{1}^{m}(E)=0 \text { implies }\|S\|(E)=0 .
$$

We have defined flat chains analytically, but Whitney introduced them first geometrically. The equivalence of the two approaches is confirmed in part by the following theorem on polyhedral approximation [23, §\$4.1.23, 4.2.24].

If $K$ is a compact subset of $U$ and

$$
S \in \mathbf{F}_{m}(U) \text { with spt } S \subset \text { Int } K,
$$

then there exist real polyhedral chains $P_{j} \in \mathbf{P}_{m}(U) \cap \mathbf{F}_{m, K}(U)$ such that

$$
\begin{gathered}
\lim _{j \rightarrow \infty} \mathbf{F}_{K}\left(P_{j}-S\right)=0, \quad \lim _{j \rightarrow \infty} \mathbf{M}\left(P_{j}\right)=\mathbf{M}(S), \\
\lim _{j \rightarrow \infty} \mathbf{M}\left(\partial P_{j}\right)=\mathbf{M}(\partial S) \quad \text { in case } m>0 .
\end{gathered}
$$

We remark that the two conclusions involving $\mathbf{M}$ would fail if in place of mass and comass we used the norms induced by the standard inner products of the Grassmann algebra of $\mathbf{R}^{n}$. (See [27, \$2].)

We gain still another pertinent view of flat chains through representation by pairs of $\mathfrak{L}^{n}$ summable multivectorfields [23, \$4.1.18]. Whenever

$$
S \in \mathbf{F}_{m, K}(U) \text { and } \varepsilon>0
$$

there exist $\mathfrak{L}^{n}$ summable functions $\xi$ and $\eta$ whose values are $m$ vectors and $m+1$ vectors of $\mathbf{R}^{n}$, respectively, such that

$$
\begin{gathered}
(\text { spt } \xi) \cup(\text { spt } \eta) \subset U \cap\{x: \operatorname{dist}(x, K) \leqslant \varepsilon\}, \\
\int(\|\xi\|+\|\eta\|) d \mathcal{L}^{n} \leqslant \mathbf{F}_{K}(S)+\varepsilon, \quad S=\left(\mathfrak{L}^{n} \wedge \xi\right)+\partial\left(\mathcal{L}^{n} \wedge \eta\right) .
\end{gathered}
$$

Of course $\xi$ and $\eta$ are not unique unless $m=n$, in which case $\eta=0$ and $\mathbf{F}_{K}(S)=\mathbf{M}(S)=\int\|\xi\| d \mathcal{L}^{n}$.

Our last description of flat chains leads immediately to Wolfe's theorem representing a locally flat cochain $\lambda \in \mathbf{F}^{m}(U)$ by a pair of locally bounded $\mathfrak{L}^{n}\llcorner U$ measurable differential forms $\phi$ and $\psi$ with degrees $m$ and $m+1$ such that

$$
\lambda\left[\left(\mathscr{L}^{n} \wedge \xi\right)+\partial\left(\mathfrak{L}^{n} \wedge \eta\right)\right]=\int_{U}(\langle\xi, \phi\rangle+\langle\eta, \psi\rangle) d \mathfrak{L}^{n}
$$

whenever $\xi$ and $\eta$ are $\mathfrak{L}^{n}$ summable $m$ and $m+1$ vectorfields with compact support in $U$, and such that (see [27, $\left.\$ 4.6(4)^{\prime}\right]$ )

$$
\mathbf{E}^{n}\left[\psi \wedge \omega+(-1)^{m} \phi \wedge d \omega\right]=0 \text { for } \omega \in \mathbb{Q}^{n-m-1}(U),
$$

which says that $\psi$ is a weak exterior derivative of $\phi$. All such pairs $(\phi, \psi)$ represent locally flat cochains. Useful sufficient conditions [27, \$6.2] are that $\phi$ be continuous $\mathcal{F}^{n-1}$ almost everywhere in $U$ and there exist a sequence of sets $F_{k}$ with $\mathcal{H}^{n-1}\left(F_{k}\right)<\infty$ for which 


$$
U \sim \bigcup_{k=1}^{\infty} F_{k} \subset\{x: x \in \operatorname{dmn} D \phi \text { and } d \phi(x)=\psi(x)\} .
$$

7. Rectifiable currents. The currents which we have discussed so far may be viewed as generalized chains with real coefficients. Now we turn to the much more significant and subtle task of extending the concept of chain with integer coefficients in ways useful for analysis. Our aim is to gain completeness and compactness properties for suitable groups of integral chains, while maintaining as much as possible of the basic geometry of sums of pieces of class one oriented manifolds with integer multiplicities. Starting with integral polyhedral chains we will use Lipschitzian maps, convergence in mass and the boundary operator to construct rectifiable currents, integral currents and integral flat chains.

Whenever $K$ is a compact subset of an open subset $U$ of $\mathbf{R}^{n}$ we define

$$
\mathcal{R}_{m, K}(U)
$$

as the class of those $m$ dimensional currents $S$ in $U$ which have the property that for every positive number $\varepsilon$ there exist a locally Lipschitzian map $f$ of an open subset $Z$ of some Euclidean space into $U$, a compact subset $C$ of $Z$ with $f(C) \subset K$, and an integral polyhedral chain

$$
P \in \mathscr{P}_{m, C}(Z) \text { with } \mathbf{M}\left(S-f_{\#} P\right)<\varepsilon .
$$

We note that

$$
\Re_{m, K}(U) \text { is an additive subgroup of } \mathbf{F}_{m, K}(U) \cap \mathbf{M}_{m}(U) .
$$

The union of the classes $\Re_{m, K}(U)$ corresponding to all compact $K \subset U$ is the group

$$
\mathcal{R}_{m}(U)
$$

of $m$ dimensional rectifiable currents in $U$.

Several alternate descriptions [23, \$4.1.28] of rectifiable currents are useful. For example, $\Re_{m}(U)$ consists of the currents

$$
\left(\mathcal{H}^{m}\llcorner W) \wedge \xi\right.
$$

corresponding to all $\left(\mathcal{F H}^{m}, m\right)$ rectifiable Borel sets $W$ with compact closure in $U$ and all $\mathcal{H}^{m}\left\llcorner W\right.$ summable $m$ vectorfields $\xi$ such that, for $\mathcal{F}^{m}$ almost all $x$ in $W$,

$\xi(x)$ is a simple $m$ vector, $\|\xi(x)\|$ is a positive integer,

$\operatorname{Tan}^{m}\left(\mathcal{H}^{m} L W, x\right)$ is the vectorspace associated with $\xi(x)$.

In fact, if $S \in \mathscr{R}_{m}(U)$, then $\|S\|=\mathcal{F}^{m}\left\llcorner\Theta^{m}(\|S\|, \cdot)\right.$ and

$$
S=\left[\mathcal{F C}^{m} L\left\{x: \Theta^{m}(\|S\|, x)>0\right\}\right] \wedge \xi
$$

with

$$
\xi(x)=\Theta^{m}(\|S\|, x) \vec{S}(x) \text { for }\|S\| \text { almost all } x .
$$

We observe that $\mathcal{H C}^{m}$ almost all of $\left\{x: \Theta^{m}(\|S\|, x)>0\right\}$ can be covered by a countable family of $m$ dimensional submanifolds of class 1 of $U$, but spt $S$ may contain a nonempty open subset of $U$. Another characterization states 
that

$$
\begin{aligned}
& S \in \Re_{m}(U) \text { if and only if } S \in \mathbf{F}_{m}(U) \cap \mathbf{M}_{m}(U) \text { and } \\
& \Theta^{m}(\|S\|, x) \text { is a positive integer for }\|S\| \text { almost all } x .
\end{aligned}
$$

(The proofs of this statement in $[50, \S 5.3 .1]$ and $[47, \S 1.4]$ adapt parts of the proof of $[23, \S 4.2 .15]$, deducing the $(\|S\|, m)$ rectifiability of $U$ from [23, \$3.3.15] and the absolute continuity of $\|S\|$ with respect to $\Phi_{1}^{m}$, then using the simple mapping properties of flat chains.)

Applying the generalized area formula to compute the image of a rectifiable current $\left(\mathcal{H}^{m}\llcorner W) \wedge \xi\right.$ under a locally Lipschitzian map one obtains

$$
f_{\#}\left[\left(\mathcal{H}^{m}\llcorner W) \wedge \xi\right]=\left[\mathcal{H}^{m}\llcorner f(W)] \wedge \eta\right.\right.
$$

with

$$
\eta(y)=\sum_{x \in W \cap f^{-1}\{y\}} \zeta(x) \text { for } \mathcal{H}^{m} \text { almost all } y,
$$

where

$$
\zeta(x)=\left[\wedge_{m} \mathrm{ap} D(f \mid W)(x)\right] \xi(x) / \operatorname{ap} J_{m}(f \mid W)(x)
$$

whenever $f \mid W$ has an $\left(\mathcal{H}^{m}, m\right)$ approximate differential of rank $m$ at $x$.

It happens often that $S \in \mathcal{R}_{m}(U)$ but $\partial S \notin \mathcal{R}_{m-1}(U)$. For instance

$$
\sum_{j=1}^{\infty}\left[-2^{-j}, 2^{-j}\right] \in \Re_{1}(\mathbf{R}), \quad \sum_{j=1}^{\infty}\left(\left[2^{-j}\right]-\left[-2^{-j}\right]\right) \notin \Re_{0}(\mathbf{R}) .
$$

However, we use the groups of rectifiable currents to construct chain complexes closed to the boundary operator in two ways, by restriction or enlargement, as follows:

The union of the groups

$$
\mathbf{I}_{m, K}(U)=\left\{S: S \in \Re_{m, K}(U), \partial S \in \Re_{m-1, K}(U) \text { or } m=0\right\}
$$

corresponding to all compact $K \subset U$ is the group

$$
\mathbf{I}_{m}(U)
$$

of $m$ dimensional integral currents in $U$.

The union of the groups

$$
\mathscr{F}_{m, K}(U)=\left\{R+\partial T: R \in \mathscr{R}_{m, K}(U), T \in \mathcal{R}_{m+1, K}(U)\right\}
$$

corresponding to all compact $K \subset U$ is the group

$$
\mathcal{F}_{m}(U)
$$

of $m$ dimensional integral flat chains in $U$.

Sometimes we need integral chains with noncompact support. Therefore we define the group

$$
\begin{aligned}
& \mathscr{R}_{m}^{\text {loc }}(U)=\mathscr{D}_{m}(U) \cap\{S: \text { For every } x \in U \text { there exists } \\
& \left.\qquad R \in \mathscr{R}_{m}(U) \text { with } x \notin \operatorname{spt}(S-R)\right\}
\end{aligned}
$$


of $m$ dimensional locally rectifiable currents in $U$, and similarly the groups $\mathbf{I}_{m}^{\text {loc }}(U)$ and $\mathscr{F}_{m}^{\text {loc }}(U)$ of locally integral currents and locally integral flat chains.

For example, if $W$ is an $m$ dimensional submanifold of class 1 of $U$, with $\mathcal{H}^{m}(W \cap K)<\infty$ for all compact $K \subset W$, and if $\xi$ is a tangent $m$ vectorfield on $W$ orienting $W$, so that $\xi$ is continuous and $\|\xi\|=1$, then the corresponding locally rectifiable current $\left(\mathcal{H}^{m} L W\right) \wedge \xi$ equals the classical operator integrating differential forms of degree $m$ over the oriented manifold $W[23, \$ 4.1 .31]$. Hence, in classical notation,

$$
\left[\left(\mathcal{H}^{m} L W\right) \wedge \xi\right](\phi)=\int_{W} \phi \text { for } \phi \in \mathscr{Q}^{m}(U) .
$$

We call $\left(\mathcal{H}^{m}\llcorner W) \wedge \xi\right.$ an orientation current of $W$.

Every 1 dimensional integral current is a sum of finitely many oriented simple arcs and countably many simple closed curves, with finite total length. Every $n$ dimensional integral current in $\mathbf{R}^{n}$ is a countable sum of currents $\pm \mathbf{E}^{n} L A$ corresponding to $\mathscr{L}^{n}$ measurable sets $A$ for which our version of the Gauss-Green formula holds. However, the structure of $m$ dimensional integral currents in $\mathbf{R}^{n}$ can be very complicated when $1<m<n[23$, \$4.2.25].

The classes of currents most important for our work appear in the diagram of inclusions:

$$
\begin{array}{lllll}
\mathbf{I}_{m, K}(U) & \subset & \Re_{m, K}(U) & \subset & \mathscr{F}_{m, K}(U) \\
\hat{\mathbf{N}}_{m, K}(U) & \subset & \mathbf{F}_{m, K}(U) \cap \mathbf{M}_{m}(U) & \subset & \mathbf{F}_{m, K}(U)
\end{array}
$$

There is an integral analogue $\mathscr{F}_{K}$ of the real flat norm $\mathbf{F}_{K}$. For $S \in$ $\mathscr{F}_{m, K}(U)$ we define

$$
\begin{aligned}
\mathscr{F}_{K}(S)=\inf \{\mathbf{M}(R)+\mathbf{M}(T): & R \in \mathscr{R}_{m, K}(U), \\
T & \left.\in \Re_{m+1, K}(U), R+\partial T=S\right\} .
\end{aligned}
$$

The group $\mathscr{F}_{m, K}(U)$ is a complete metric space with respect to the integral flat distance $\mathscr{F}_{K}\left(S_{1}-S_{2}\right)$ between $S_{1}$ and $S_{2}$. Moreover, $\mathbf{I}_{m, K}(U)$ is $\mathscr{F}_{K}$ dense in $\mathscr{F}_{m, K}(U)$. Since Lipschitzian deformation chains of rectifiable currents are rectifiable, one can use the homotopy formula to estimate the integral flat distance between the images of an integral current under Lipschitz-homotopic maps.

A close connection between integral flat chains and elementary geometry is expressed by the following approximation theorem [23, \$\$4.2.21, 4.2.22]:

If $K$ is a compact subset of $U$ and

$$
S \in \mathscr{F}_{m}(U) \text { with spt } S \subset \text { Int } K,
$$

then there exist integral polyhedral chains $P_{j} \in \mathscr{P}_{m}(U) \cap \mathscr{F}_{m, K}(U)$ such that

$$
\begin{gathered}
\lim _{j \rightarrow \infty} \mathscr{F}_{K}\left(P_{j}-S\right)=0, \quad \lim _{j \rightarrow \infty} \mathbf{M}\left(P_{j}\right)=\mathbf{M}(S), \\
\lim _{j \rightarrow \infty} \mathbf{M}\left(\partial P_{j}\right)=\mathbf{M}(\partial S) \quad \text { in case } m>0 .
\end{gathered}
$$

For an integral current $S$ in $U$ we can even do much better, by deforming 
$U$ slightly in such a way as to map $S$ and $\partial S$ onto currents largely overlapping an integral polyhedral chain and its boundary [23, \$4.2.20]:

Whenever $S \in \mathbf{I}_{m}(U)$ and $\varepsilon>0$ there exist

$$
P \in \mathscr{P}_{m}(U) \text { with spt } P \subset\{x: \operatorname{dist}(x, \operatorname{spt} S) \leqslant \varepsilon\}
$$

and a diffeomorphism $f$ of class 1 mapping $U$ onto $U$ such that

$$
\begin{aligned}
& \mathbf{N}\left(P-f_{\#} S\right) \leqslant \varepsilon, 1+\varepsilon \text { is a Lipschitz constant for } f \text { and } f^{-1}, \\
& |f(x)-x| \leqslant \varepsilon \text { for } x \in U, f(x)=x \text { if } \operatorname{dist}(x, \operatorname{spt} S) \geqslant \varepsilon .
\end{aligned}
$$

It follows that $\mathbf{N}\left(f_{\#}^{-1} P-S\right) \leqslant(1+\varepsilon)^{m} \varepsilon$ and $f_{\#}^{-1} P$ is a nonsingular chain of class 1 , belonging to some triangulation of class 1 of $U$.

To proceed further we need the concept of (local) Lipschitz neighborhood retract in $\mathbf{R}^{n}$, which means a set $A$ such that for some neighborhood $Z$ of $A$ in $\mathbf{R}^{n}$ there exists a (locally) Lipschitzian map

$$
f: Z \rightarrow A \text { with } f(x)=x \text { for } x \in A \text {. }
$$

Examples are convex subsets of $\mathbf{R}^{n}$, linearly embedded compact polyhedra, submanifolds of class 1 , the closures of open subsets bounded by $n-1$ dimensional submanifolds of class 1 . In case $A$ is connected, the retraction $f$ may be required to have class $k \geqslant 1$ if and only if $A$ is a submanifold of class $k[23, \S \S 4.1 .16,3.1 .20,4.1 .29]$.

The following theorem [23, $\$ 4.2 .16,4.2 .17]$ conveys the most essential information about closure and compactness properties of integral and normal currents, and about the structure of integral flat chains:

If $K$ is a compact Lipschitz neighborhood retract in $U$ and $c \in \mathbf{R}$, then

(1) $\mathbf{I}_{m, K}(U)$ is $\mathbf{F}_{K}$ closed in $\mathbf{N}_{m, K}(U)$,

(2) $\Re_{m+1, K}(U) \cap\{T: \mathbf{M}(\partial T)<\infty\}=\mathbf{I}_{m+1, K}(U)$,

(3) $\mathscr{F}_{m, K}(U) \cap\{S: \mathbf{M}(S)<\infty\}=\Re_{m, K}(U)$,

(4) $\mathbf{N}_{m, K}(U) \cap\{S: \mathbf{N}(S) \leqslant c\}$ is $\mathbf{F}_{K}$ compact,

(5) $\mathbf{I}_{m, K}(U) \cap\{S: \mathbf{N}(S) \leqslant c\}$ is $\mathscr{F}_{K}$ compact.

These results, particularly proposition (5), are basic for the new geometric methods in the calculus of variations.

The need for the flat norm in proposition (1) is illustrated by the fact that

$$
\lim _{j \rightarrow \infty} \sum_{k=1}^{j}[(2 k-1) /(2 j), k / j]=[0,1] / 2
$$

with respect to the weak topology in $\mathscr{D}_{1}(\mathbf{R})$, but of course not with respect to $\mathbf{F}_{\{x: 0 \leqslant x \leqslant 1\}}$.

In case $0<m<n$ it is not known whether $\mathscr{F}_{m, K}(U)$ is $\mathbf{F}_{K}$ closed in $\mathbf{F}_{m, K}(U)$, or whether $\nu \mathscr{F}_{m, K}(U)$ is $\mathscr{F}_{K}$ closed in $\mathscr{F}_{m, K}(U)$ for every integer $\nu$, even when $K$ is a cube.

Our measuretheoretic methods can also be used to construct [23, §4.2.26] a theory of chains with coefficients in the cyclic group $\mathbf{Z}_{\nu}=\mathbf{Z} / \nu \mathbf{Z}$ of order $\nu$. Defining the group of $m$ dimensional flat chains modulo $\nu$ as the quotient of $\mathscr{F}_{m}(U)$ by the closure of $\nu \mathscr{F}_{m}(U)$, and introducing appropriate notions of 
boundary, support, mass and rectifiability, one finds that flat chains modulo $\nu$ have structural and compactness properties largely analogous to those of integral flat chains. One can represent $m$ dimensional flat chains modulo $\nu$ with finite mass by rectifiable currents whose densities do not exceed $\nu / 2$; in case $\nu=2$ such chains correspond simply to $\left(\mathcal{H}^{m}, m\right)$ rectifiable sets with compact closure in $U$.

Many problems in classical geometry involve currents supported by analytic varieties. To each finite set $F$ of real valued analytic functions on an open subset $V$ of $\mathbf{R}^{n}$ corresponds the analytic subvariety

$$
V \cap\{x: f(x)=0 \text { whenever } f \in F\}
$$

of $V$. We call $S$ an $m$ dimensional analytic chain in $U$ if and only if

$$
S \in \mathscr{F}_{m}^{\mathrm{doc}}(U)
$$

and every point of $U$ has a neighborhood $V$ with analytic subvarieties $A, B$ such that

$$
\begin{gathered}
\operatorname{dim} A<m \text { and } V \cap \operatorname{spt} S \subset A, \\
\operatorname{dim} B<m-1 \text { and } V \cap \operatorname{spt} \partial S \subset B \text { or } m=0 .
\end{gathered}
$$

Local analytic geometry [23, $\$ 3.4 .5-12,4.2 .28-29]$ has the following implications:

All $m$ dimensional analytic chains in $U$ are locally integral currents, and are locally representable as finite sums of integral multiples of disjoint oriented $m$ dimensional analytic submanifolds of $U$.

If $P$ and $Q$ are analytic subvarieties of $U, \operatorname{dim}(P \sim Q)=m$,

$$
R=\bigcup\{V \cap(P \sim Q): V \text { is open in } U \text { and } V \cap(P \sim Q)
$$

is an $m$ dimensional analytic submanifold of $V$ \}

is the regular part of $P \sim Q$, and if $W$ is a component of $R$ which can be oriented by a continuous tangent $m$ vectorfield $\xi$ with $|\xi|=1$, then $\left(\mathcal{H}^{m} L W\right) \wedge \xi$ is an $m$ dimensional analytic chain in $U$.

Similarly one uses complex valued holomorphic functions to define holomorphic subvarieties of $\mathbf{C}^{n}$. We call $S$ a complex $\kappa$ dimensional holomorphic chain in an open subset $U$ of $\mathbf{C}^{n}$ if and only if

$$
S \in \mathscr{F}_{2 \kappa}^{\circ}(U), \partial S=0 \text { or } \kappa=0,
$$

and every point of $U$ has a neighborhood $V$ with a holomorphic subvariety $A$ such that

$$
\operatorname{dim}_{\mathrm{C}} A<\kappa \text { and } V \cap \operatorname{spt} S \subset A .
$$

It follows that $S$ is locally rectifiable. We say that $S$ is positive in case for $\|S\|$ almost all $z$ there exists a factorization

$$
\vec{S}(z)=v_{1} \wedge i v_{1} \wedge \cdots \wedge v_{\kappa} \wedge i v_{\kappa} \text { with } v_{1}, \ldots, v_{\kappa} \in \mathbf{C}^{n} .
$$

If $\boldsymbol{P}$ is a complex $\kappa$ dimensional holomorphic subvariety of $U$, then the regular part $R$ of $P$ is oriented by a unique positive $2 \kappa$ vectorfield $\xi$, and $\left(\mathcal{H}^{2 \kappa} L R\right) \wedge \xi$ is the unique complex $\kappa$ dimensional positive holomorphic chain $S$ in $U$ for which $\|S\|=\mathcal{H}^{2 \kappa} L P$. 
8. Homology groups and isoperimetric inequalities. We will employ the local Lipschitz category, whose objects are the pairs $(A, B)$ such that, for some $n, A$ and $B$ are local Lipschitz neighborhood retracts in $\mathbf{R}^{n}$ with $B \subset A$, and whose morphisms are the locally Lipschitzian maps

$$
f:(A, B) \rightarrow\left(A^{\prime}, B^{\prime}\right) .
$$

We define the groups of $m$ dimensional integral flat chains, cycles, boundaries

$$
\begin{aligned}
\mathscr{F}_{m}(A) & =\mathscr{F}_{m}\left(\mathbf{R}^{n}\right) \cap\{S: \text { spt } S \subset A\}, \\
\mathscr{Z}_{m}(A, B) & =\mathscr{F}_{m}(A) \cap\left\{S: \partial S \in \mathscr{F}_{m-1}(B) \text { or } m=0\right\}, \\
\mathscr{B}_{m}(A, B) & =\left\{R+\partial T: R \in \mathscr{F}_{m}(B), T \in \mathscr{F}_{m+1}(A)\right\}
\end{aligned}
$$

and the integral homology groups

$$
\mathbf{H}_{m}(A, B ; \mathbf{Z})=\mathscr{Z}_{m}(A, B) / \mathscr{B}_{m}(A, B) .
$$

Replacement of $\mathbf{R}^{n}$ by any neighborhood of $A$ in $\mathbf{R}^{n}$ yields isomorphic groups. Each locally Lipschitzian function on $A$ can be extended to some neighborhood retractible onto $A$, and any such extension yields chain homomorphisms which in turn induce homology homomorphisms

$$
\mathbf{H}_{m}(f ; \mathbf{Z}): \mathbf{H}_{m}(A, B ; \mathbf{Z}) \rightarrow \mathbf{H}_{m}\left(A^{\prime}, B^{\prime} ; \mathbf{Z}\right) .
$$

Moreover, the boundary operator induces connecting transformations

$$
\partial_{m}(A, B ; \mathbf{Z}): \mathbf{H}_{m}(A, B ; \mathbf{Z}) \rightarrow \mathbf{H}_{m-1}(B ; \mathbf{Z}) .
$$

One verifies quite easily that these functors and natural transformations satisfy the axioms of Eilenberg and Steenrod for a homology theory with coefficient group $\mathbf{Z}$. Regarding excision one finds that inclusion maps

$$
\begin{gathered}
(A, B) \hookrightarrow\left(A^{\prime}, B^{\prime}\right) \quad \text { with } A \cap B^{\prime}=B, \\
A^{\prime} \cap \operatorname{Clos}\left(A^{\prime} \sim B^{\prime}\right) \subset A, \quad A^{\prime} \cap \operatorname{Clos}\left(A^{\prime} \sim A\right) \subset B^{\prime}
\end{gathered}
$$

induce homology isomorphisms [23, \$4.4.1].

Our homology theory is isomorphic with the restriction of the classical singular theory to the local Lipschitz category. However, for problems involving integration and for intersection theory, our chain groups are decisively preferable to the singular chain groups. It should be noted that flat chains, unlike singular chains, are equal to their subdivisions. This identification simplifies the construction of cycles, and yields better cocycles with real coefficients. On the other hand it is an open question how usefully integral flat chains could be related to cohomology with integer coefficients.

One of the turning points in the development of geometric measure theory was the discovery that isoperimetric inequalities do not only apply to certain special situations studied in classical differential geometry, but are generally valid metric estimates related to the homology theory of arbitrary pairs in the local Lipschitz category. This fundamental connection between measure theory and algebraic topology is described by the following theorem [23, 84.4.2].

Suppose $(A, B)$ belongs to the local Lipschitz category, $K$ is a compact subset of $A$, and $K \cap B$ is compact. 
(1) One can find a positive number $\omega$ such that

$$
\mathfrak{Z}_{m}(A, B) \cap\{S: \text { spt } S \subset K, \mathbf{M}(S) \leqslant \omega\} \subset \Re_{m}(A, B) .
$$

(2) One can find a compact subset $K^{\prime}$ of $A$ and a real number $\sigma$ such that for every $S \in \mathscr{B}_{m}(A, B)$ with spt $S \subset K$ there exists

$$
\begin{gathered}
T \in \mathscr{F}_{m+1}(A) \text { with spt } T \subset K^{\prime}, \operatorname{spt}(S-\partial T) \subset B, \\
{[\mathbf{M}(T)]^{m /(m+1)}+\mathbf{M}(S-\partial T) \leqslant \sigma \mathbf{M}(S) .}
\end{gathered}
$$

It follows that $\mathscr{B}_{m}(A, B) \cap \mathscr{F}_{m, K}\left(\mathbf{R}^{n}\right)$ is relatively open and closed in $\mathscr{Z}_{m}(A, B) \cap \mathscr{F}_{m, K}\left(\mathbf{R}^{n}\right)$ with respect to $\mathscr{F}_{K}$.

Combining this isoperimetric theorem with the compactness theorem for $\mathbf{N}$ bounded sets of integral currents one can prove that for every compact Lipschitz neighborhood retract $K$ contained in $A$ and each $c \in \mathbf{R}$ the two sets

$$
\begin{aligned}
& \mathscr{Z}_{m}(A, B) \cap\{S: \text { spt } S \subset K, \mathbf{M}(S) \leqslant c\}, \\
& \mathscr{B}_{m}(A, B) \cap\{S: \operatorname{spt} S \subset K, \mathbf{M}(S) \leqslant c\}
\end{aligned}
$$

are $\mathscr{F}_{K}$ compact in case $B \cap K=\varnothing$, and are $\mathscr{F}_{K, B}$ compact in case $B \cap K \neq$ $\varnothing$, where

$$
\mathscr{F}_{K, B}(S)=\int_{0}^{* \infty} \mathscr{F}_{K}\left[S\llcorner\{x: \operatorname{dist}(x, K \cap B)>r\}] d \mathscr{L}^{\mathbf{l}} r\right.
$$

whenever $S \in \mathcal{R}_{m, K}\left(\mathbf{R}^{n}\right)$. These facts are applied in the solution of variational problems with homological constraints.

Replacing integral flat chains by flat chains modulo $\nu$, one obtains analogous results for a measuregeometric homology theory with coefficient group $\mathbf{Z}_{\nu}$.

In the case of the real coefficient field $\mathbf{R}$ the situation is only partially analogous. Proceeding as before we define the vectorspaces of $m$ dimensional real flat chains, cycles, boundaries

$$
\begin{gathered}
\mathbf{F}_{m}(A)=\mathbf{F}_{m}\left(\mathbf{R}^{n}\right) \cap\{S: \text { spt } S \subset A\}, \\
\mathbf{Z}_{m}(A, B)=\mathbf{F}_{m}(A) \cap\left\{S: \partial S \in \mathbf{F}_{m}(B) \text { or } m=0\right\}, \\
\mathbf{B}_{m}(A, B)=\left\{R+\partial T: R \in \mathbf{F}_{m}(B), T \in \mathbf{F}_{m+1}(A)\right\},
\end{gathered}
$$

the homology vectorspaces with real coefficients

$$
\mathbf{H}_{m}(A, B ; \mathbf{R})=\mathbf{Z}_{m}(A, B) / \mathbf{B}_{m}(A, B),
$$

the induced homomorphisms $\mathbf{H}_{m}(f ; R)$ and the connecting transformations $\partial_{m}(A, B ; \mathbf{R})$. Thus we obtain a homology theory with coefficient group $\mathbf{R}$ on the local Lipschitz category, which is isomorphic to the restriction of the classical real singular theory. However, the real analogue of the integral isoperimetric theorem is false. On a circle there are 1 dimensional real cycles with arbitrarily small mass which do not bound. For 2 dimensional normal currents $T$ in $\mathbf{R}^{2}$ (which are uniquely determined by $\partial T$ ) the ratios

$$
\mathbf{M}(T) /[\mathbf{M}(\partial T)]^{2}
$$

can be arbitrarily large [23, §4.5.13]. Only the following modified estimate is true [27, \$3.1]. 
Suppose $(A, B)$ belongs to the local Lipschitz category, $K$ is a compact subset of $A$, and $K \cap B$ is compact.

One can find a compact subset $K^{\prime}$ of $A$ and a real number $\tau$ such that for every $S \in \mathbf{B}_{m}(A, B)$ with spt $S \subset K$ there exists

$$
\begin{gathered}
T \in \mathbf{F}_{m+1}(A) \quad \text { with spt } T \subset K^{\prime}, \operatorname{spt}(S-\partial T) \subset B, \\
\mathbf{M}(T)+\mathbf{M}(S-\partial T) \leqslant \tau \mathbf{M}(S) .
\end{gathered}
$$

When $B$ is relatively closed in $A$ it follows that

$$
\mathbf{B}_{m}(A, B) \cap \mathbf{F}_{m, K}\left(\mathbf{R}^{n}\right) \text { is } \mathbf{F}_{K} \text { closed in } \mathbf{F}_{m, K}\left(\mathbf{R}^{n}\right) \text {; }
$$

moreover $\mathbf{Z}_{m}(A, B)$ and $\mathbf{B}_{m}(A, B)$ have $\mathbf{M}$ bounded compactness properties analogous to those of $\mathscr{Z}_{m}(A, B)$ and $\mathscr{B}_{m}(A, B)$, with respect to $\mathrm{F}_{K}$ and the function $\mathbf{F}_{K, B}$ analogous to $\mathscr{F}_{K, B}$, provided $K$ is a Lipschitz neighborhood retract.

The flat closedness of $\mathbf{B}_{m}(A, B)$ is useful not only in homological variational problems but also in the proof of the following generalizations [27, $\S \S 4.8,4.9]$ of two classical theorems of De Rham, which provide an isomorphism between

$$
\operatorname{Hom}_{\mathbf{R}}\left[\mathbf{H}_{m}(A, B ; \mathbf{R}), \mathbf{R}\right]
$$

and the $m$ th cohomology group of the complex of germs of smooth differential forms defined on neighborhoods of $A$ and vanishing on neighborhoods of $B$.

Suppose $(A, B)$ belongs to the local Lipschitz category and $B$ is relatively closed in $A$.

(1) $A$ and $B$ have neighborhoods $U$ and $V$ such that for every $\mathbf{R}$ linear map

$$
v: \mathbf{H}_{m}(A, B ; \mathbf{R}) \rightarrow \mathbf{R}
$$

there exists

$$
\begin{gathered}
\phi \in \mathcal{E}^{m}(U) \quad \text { with } d \phi=0, \text { spt } \phi \subset U \sim V, \\
S(\phi)=v(\chi) \quad \text { whenever } S \in \chi \in \mathbf{H}_{m}(A, B ; \mathbf{R}) .
\end{gathered}
$$

(2) Given any neighborhoods $U$ and $V$ of $A$ and $B$, one can find neighborhoods $U^{\prime}$ and $V^{\prime}$ of $A$ and $B$ such that for every

$$
\begin{gathered}
\phi \in \mathcal{E}^{m}(U) \text { with } m>0, d \phi=0, \text { spt } \phi \subset U \sim V, \\
S(\phi)=0 \text { whenever } S \in \mathbf{Z}_{m}(A, B),
\end{gathered}
$$

there exists

$$
\psi \in \mathcal{E}^{m-1}\left(U^{\prime}\right) \text { with } d \psi=\phi \mid U^{\prime}, \text { spt } \psi \subset U^{\prime} \sim V^{\prime} .
$$

9. Parametric integrals. Our discusion of $m$ dimensional surface integrals has been confined so far to the case where the integrand corresponds to a differential form of degree $m$. Now we consider an arbitrary parametric integrand $\Psi$ of degree $m$ on the open subset $U$ of $\mathbf{R}^{n}$. This is, by definition, a continuous map 
which satisfies the homogeneity condition

$$
\Psi(x, t \xi)=t \Psi(x, \xi) \text { for } x \in U, \xi \in \wedge_{m} \mathbf{R}^{n}, 0<t \in \mathbf{R} .
$$

We call $\Psi$ a positive integrand if and only if

$$
\Psi(x, \xi)>0 \text { for } x \in U, 0 \neq \xi \in \wedge_{m} \mathbf{R}^{n} .
$$

We term $\Psi$ an integrand of class $k$ if and only if the restriction of $\Psi$ to $U \times\left(\bigwedge_{m} \mathbf{R}^{n} \sim\{0\}\right)$ is a function of class $k$.

Examples of positive integrands are

the area integrand mapping $(x, \xi)$ onto $|\xi|=(\xi \bullet \xi)^{1 / 2}$,

where - designates the inner product of $\bigwedge_{m} \mathbf{R}^{n}$ induced by the standard inner product of $\mathbf{R}^{n}$, and

$$
\text { the mass integrand mapping }(x, \xi) \text { onto }\|\xi\| \text {. }
$$

Similar integrands are associated with every Riemannian metric tensor on $U$.

For $S \in \mathbf{M}_{m}(U)$ we define the parametric integral

$$
\int_{S} \Psi=\int \Psi[x, \vec{S}(x)] d\|S\| x .
$$

The homogeneity property of $\Psi$ implies

$$
\int_{\gamma \wedge \zeta} \Psi=\int \Psi[x, \zeta(x)] d \gamma x
$$

for every Radon measure $\gamma$ with compact support in $U$ and every $\gamma$ summable $m$ vectorfield $\zeta$. We also note that

$$
\begin{gathered}
\int_{S} \Psi=S(\phi) \quad \text { in case } S \in \mathbf{M}_{m}(U), \phi \in \mathcal{E}^{m}(U), \\
\Psi(x, \xi)=\langle\xi, \phi(x)\rangle \text { for } x \in U, \xi \in \wedge_{m} \mathbf{R}^{n},
\end{gathered}
$$

and that $\int_{S} \Psi=\mathbf{M}(S)$ in case $\Psi$ is the mass integrand.

Certain pointwise properties of parametric integrands are linked to convergence properties of the corresponding integrals [27, \$2]. First we state a proposition relating convexity of a positive integrand to lowersemicontinuity of the integral on the weakly topologized space of currents with finite mass.

For each positive integrand $\Psi$ on $U$ the following three conditions are equivalent:

(1) $\Psi$ is a convex integrand; this means that

$$
\Psi(x, \xi+\eta)<\Psi(x, \xi)+\Psi(x, \eta) \text { for } x \in U \text { and } \xi, \eta \in \wedge_{m} \mathbf{R}^{n} \text {. }
$$

$$
\int_{S} \Psi=\sup \left\{S(\phi): \Psi \geqslant \phi \in \mathscr{D}^{m}(U)\right\}
$$

whenever $S \in \mathbf{M}_{m}(U)$; here $\Psi \geqslant \phi$ means that

$$
\begin{gathered}
\Psi(x, \xi)>\langle\xi, \phi(x)\rangle \text { for } x \in U, \xi \in \wedge_{m} \mathbf{R}^{n} . \\
\liminf _{j \rightarrow \infty} \int_{R_{,}} \Psi>\int_{S} \Psi
\end{gathered}
$$


whenever all $R_{j}$ and $S$ belong to $\mathbf{M}_{m}(U)$ with

$$
\lim _{j \rightarrow \infty} R_{j}(\phi)=S(\phi) \quad \text { for } \phi \in \mathscr{D}_{m}(U) .
$$

In case $H$ is a submanifold of class 1 of $U$, we say that $\Psi$ is $H$ flat if and only if, for $x \in H$ and $\xi \in \wedge_{m} \operatorname{Tan}(H, x), \Psi(x, \xi)$ is nonnegative and equals the infimum of the set of numbers

$$
\sum_{i=1}^{N} \Psi\left(x, \eta_{i}\right)
$$

corresponding to all finite sequences of simple $m$ vectors $\eta_{1}, \ldots, \eta_{N}$ in $\bigwedge_{m} \operatorname{Tan}(H, x)$ such that

$$
\xi=\sum_{i=1}^{N} \eta_{i}
$$

For the mass integrand this condition holds always, but for the area integrand it fails when $1<m<(\operatorname{dim} H)-1$.

Flatness of a positive integrand is a pointwise property related, in case $H=U$, to lowersemicontinuity and polyhedral approximation of the integral on the space of real flat chains with finite mass.

$A$ positive integrand $\Psi$ is $U$ flat if and only if the following two conditions hold for each compact $K \subset U$ :

$$
\liminf _{j \rightarrow \infty} \int_{R_{j}} \Psi>\int_{S} \Psi
$$

whenever all $R_{j}$ and $S$ belong to $\mathbf{F}_{m, K}(U) \cap \mathbf{M}_{m}(U)$ with

$$
\lim _{j \rightarrow \infty} \mathbf{F}_{K}\left(R_{j}-S\right)=0 .
$$

(II) For every $S \in \mathbf{F}_{m, K}(U) \cap \mathbf{M}_{m}(U)$ with spt $S \subset$ Int $K$ there exist $P_{j} \in$ $\mathbf{P}_{m}(U)$ with spt $P_{j} \subset$ Int $K$ such that

$$
\lim _{j \rightarrow \infty} \mathbf{F}_{K}\left(P_{j}-S\right)=0 \text { and } \lim _{j \rightarrow \infty} \int_{P_{j}} \Psi=\int_{S} \Psi \text {. }
$$

Next we will define the notion of ellipticity for a parametric integrand. This pointwise property plays a crucial role in the regularity theory for rectifiable currents minimizing a parametric integral.

We say that $\Psi$ is elliptic at the point $a$ in $U$, with ellipticity bound $c>0$, if and only if

$$
\int \Psi[a, \vec{R}(x)] d\|R\| x-\int \Psi[a, \vec{S}(x)] d\|S\| x>c[\mathbf{M}(R)-\mathbf{M}(S)]
$$

whenever $R \in \Re_{m}\left(\mathbf{R}^{n}\right), S \in \Re_{m}\left(\mathbf{R}^{n}\right), \partial R=\partial S$, spt $S$ is contained in the vectorsubspace of $\mathbf{R}^{n}$ associated with a simple $m$ vector $\xi$ of $\mathbf{R}^{n}$ and

$$
\vec{S}(x)=\xi \text { for }\|S\| \text { almost all } x \text {. }
$$

We call $\Psi$ an elliptic integrand if and only if $\Psi$ is elliptic at each point of $U$ and for each compact $K \subset U$ there exists a common ellipticity bound at all points of $K$. 
Ellipticity of $\Psi$ at $a$ with bound $c$ is implied [28, 83] by the Weierstrass condition

$$
\Psi(a, \eta)-\langle(0, \eta), D \Psi(a, \xi)\rangle \geqslant c|\eta-\xi|^{2} / 2
$$

for all simple $m$ vectors $\xi, \eta$ of $\mathbf{R}^{n}$ with $|\xi|=|\eta|=1$.

Replacement of the positive ellipticity bound $c$ by 0 leads to the notion of semielliptic integrand. This pointwise property is related to lowersemicontinuity of the integral on the group of rectifiable currents.

A positive integrand $\Psi$ is semielliptic if and only if, for each compact $K \subset U$,

$$
\liminf _{j \rightarrow \infty} \int_{R_{j}} \Psi \geqslant \int_{S} \Psi
$$

whenever all $R_{j}$ and $S$ belong to $\Re_{m, K}(U)$ with

$$
\lim _{j \rightarrow \infty} \mathscr{F}_{K}\left(R_{j}-S\right)=0 .
$$

(Half of this equivalence was proved in [23, \$5.1.5]. Regarding the other half we observe that if semiellipticity fails, then it fails in case $S$ is some integral multiple of an $m$ dimensional oriented cube, which equals the sum of arbitrarily small similar cubes, for whose multiples semiellipticity fails homothetically.)

10. Minimizing currents. Assuming that $(A, B)$ belongs to the local Lipschitz category, $B$ is relatively closed in $A$ and $\Psi$ is a positive convex parametric integrand of degree $m$ on a neighborhood $U$ of $A$ in $\mathbf{R}^{n}$, such that there exists a locally Lipschitzian retraction of $U$ onto $A$, we now consider the problem of minimizing the integral of $\Psi$ on certain sets of currents with finite mass. We define

$$
\Psi_{\Gamma}(S)=\inf \left\{\int_{Q} \Psi: Q \in \mathbf{M}_{m}(U), Q-S \in \Gamma\right\}
$$

whenever $\Gamma \subset \mathbf{F}_{m}(A), 0 \in \Gamma, \Gamma+\Gamma=\Gamma$ and $S \in \mathbf{F}_{m}(A)$.

To convey the purpose of this concept we offer three initial illustrations:

If $S \in \mathscr{F}_{m}(A)$, then

$$
\Psi_{\mathbb{Z}_{m}(A, B)}(S)
$$

equals the infimum of the integrals

$$
\int_{Q\llcorner(A \sim B)} \Psi
$$

corresponding to all $m$ dimensional rectifiable currents $Q$ with

$$
\text { spt } Q \subset A \text { and } \operatorname{spt}(\partial Q-\partial S) \subset B \text {. }
$$

In case $B=\varnothing$ we thus formulate in terms of rectifiable currents the classical minimum problem with prescribed boundary $\partial S$. In case $B \neq \varnothing$ we allow a partially free boundary in $B$.

If $S \in \chi \in \mathbf{H}_{m}(A, B ; \mathbf{Z})$, then

$$
\Psi_{\mathscr{B}_{m}(A, B)}(S)=\inf \left\{\int_{Q} \Psi: Q \in \chi \cap \mathbf{M}_{m}(U)\right\}
$$


If $A=\mathbf{R}^{2} \cap\{x:|x| \geqslant 1\}, p \in A, q \in A$ and $S$ is a curve in $A$ from $p$ to $q$ which loops $k$ times around the origin, then

$$
\Psi_{\mathfrak{B}_{1}(A, \varnothing)}(S)
$$

equals the infimum of the integrals $\int_{Q} \Psi$ corresponding to all those one dimensional rectifiable currents $Q$ in $\boldsymbol{A}$ with boundary $[q]-[p]$ which loop $k$ times around the origin.

The preceding examples involve chains with coefficients in $\mathbf{Z}$. By appropriate choices of $S$ and $\Gamma$ one formulates analogous problems for chains with coefficients in $\mathbf{R}$, or in $\mathbf{Z}_{\boldsymbol{v}}$.

In many important cases one can prove the existence of a current

$$
Q \in \mathbf{M}_{m}(U) \text { with } Q-S \in \Gamma \text { and } \Psi_{\Gamma}(S)=\int_{Q} \Psi,
$$

and then $Q$ has the property that

$$
\Psi_{\Gamma}\left(Q\llcorner E)=\int_{Q\llcorner E} \Psi \text { for every compact } E \subset A .\right.
$$

An $m$ dimensional locally flat chain $Q$ with (possibly noncompact) support in $A$, which is representable by integration and has the preceding property, is called $\Psi$ minimizing with respect to $\Gamma$. In some special circumstances this property implies that the set

$$
\text { spt } Q \sim(B \cup \operatorname{spt} \partial Q)
$$

is a smooth $m$ dimensional submanifold of $U$, but often this set has singularities, even if $A=\mathbf{R}^{n}$. Also, the minimizing property of some currents can be detected by their values for suitable differential forms. We now proceed to a systematic account of what is known about such questions.

Combining the compactness and closure properties of the groups of integral flat cycles and boundaries with the lowersemicontinuity of $\Psi$ integration one obtains $[23,85.1 .6]$ the following general theorem on the existence of $\Psi$ minimizing rectifiable currents:

If $K$ is a compact subset of $A$,

$$
\begin{aligned}
& \mathscr{W}_{m, K}(A, B)=\mathscr{I}_{m}(A, B) \cap\{R: \text { spt } R \subset K\}, \\
& \mathscr{B}_{m, K}(A, B)=\mathscr{O}_{m}(A, B) \cap\{R: \text { spt } R \subset K\}
\end{aligned}
$$

and $S \in \mathscr{F}_{m, K}(U)$, then there exist $m$ dimensional rectifiable currents $Q, Q^{\prime}$ such that

$$
\begin{gathered}
Q-S \in \mathscr{Z}_{m, K}(A, B) \quad \text { and } \quad \Psi_{\mathscr{I}_{m, K}(A, B)}(S)=\int_{Q} \Psi \\
Q^{\prime}-S \in \mathscr{P}_{m, K}(A, B) \text { and } \quad \Psi_{\mathscr{S}_{m, K}(A, B)}(S)=\int_{Q^{\prime}} \Psi .
\end{gathered}
$$

We refer to $Q$ (or $Q^{\prime}$ ) as absolutely (or homologically) $\Psi$ minimizing in $K$ with respect to $(A, B)$ over $Z$.

Simple examples, like the length integrand on a punctured plane, explain the need for restriction to a compact subset of $A$. 
The first half of our existence theorem includes a solution of the problem of Plateau. The second half implies, in case $A$ is compact, that for every homology class

$$
\chi \in \mathbf{H}_{m}(A, B ; \mathbf{Z})
$$

there exists a (relative) cycle $Q^{\prime} \in \chi$ for which $\int_{Q^{\prime}} \Psi$ is least. Thom discovered a smooth 14 dimensional manifold $A$ with a homology class $\chi \in \mathbf{H}_{7}(A, \varnothing ; \mathbf{Z})$ such that $\chi$ is not the image of the fundamental class of any 7 dimensional compact oriented manifold $N$ under a continuous map of $N$ into $A$; in this case the support of a $\Psi$ minimizing cycle $Q^{\prime} \in \chi$ cannot be a manifold. Also, Federer proved that every complex $\kappa$ dimensional holomorphic subvariety of an open subset $A$ of $\mathbf{C}^{n}$ is the support of a $2 \kappa$ dimensional locally rectifiable current which is mass minimizing with respect to $\mathscr{Z}_{2 \kappa}(A, \varnothing)$; often the singular part of such a variety has complex dimension $\kappa-1$. These and other examples illustrate the immense difficulties presented by the problem of structurally characterizing the supports of minimizing rectifiable currents. Nevertheless, very significant partial results have been obtained.

Interior structure (or regularity) theory deals with a minimizing rectifiable current $Q$ in a smooth manifold $A$ and investigates local properties of spt $Q$ in the complement of $B \cup \mathrm{spt} \partial Q$. Clearly it suffices to prove regularity properties of spt $Q$ for the special case when $A$ is an open subset of $\mathbf{R}^{n}$,

$$
U=A, Q \in \mathcal{R}_{m}^{\text {loc }}(A), \partial Q=0
$$

and $Q$ is $\Psi$ minimizing with respect to $\mathcal{Z}_{m}(A, \varnothing)$. Beginning with the observation that, for $\|Q\|$ almost all $x$,

$$
\begin{gathered}
\text { Tan(spt } Q, x) \text { is an } m \text { dimensional vectorspace } \\
\text { and } \Theta^{m}(\|Q\|, x) \text { is a positive integer, }
\end{gathered}
$$

one tries to prove that the tangent spaces vary continuously on a large relatively open subset of spt $Q$. Then higher order smoothness of such a part of spt $Q$ follows from Morrey's regularity theory for the solutions of classical nonparametric variational problems. Among the connected relatively open subsets of spt $Q$, the smooth $m$ dimensional manifolds appear to be those sets on which the density of $\|Q\|$ is constant. For the validity of regularity theorems, convexity of the integrand does not suffice, but ellipticity does. After earlier work by De Giorgi and Reifenberg on the special case of the area integrand, Almgren developed a general method which led to the following theorem [23, \$5.3.16].

If $\Psi$ is a positive elliptic integrand of degree $m$ and class $k+1 \geqslant 3$ on the open subset $A$ of $\mathbf{R}^{n}$,

$$
Q \in \mathcal{R}_{m}^{\text {loc }}(A), \quad \partial Q=0,
$$

$Q$ is $\Psi$ minimizing with respect to $\mathscr{\Xi}_{m}(A, \varnothing)$,

$a \in \operatorname{spt} Q, \operatorname{Tan}^{m}(\|Q\|, a)$ is contained in some $m$ dimensional vectorsubspace of $\mathbf{R}^{n}$, and $a$ has a neighborhood $V$ in $A$ such that

$$
\Theta^{m}(\|Q\|, x) \geqslant \Theta^{* m}(\|Q\|, a) \text { for }\|Q\| \text { almost all } x \text { in } V,
$$


then a has a neighborhood $W$ in $A$ such that $W \cap \operatorname{spt} Q$ is an $m$ dimensional submanifold of class $k$ of $A$.

A point $a \in$ spt $Q$ is termed regular or singular with respect to $Q$ according to whether or not $a$ has a neighborhood $W$ in $A$ such that $W \cap \operatorname{spt} Q$ is an $m$ dimensional submanifold of class 2 of $A$.

Since the points $a$ satisfying the hypotheses of the preceding theorem form a dense subset of spt $Q$, we infer the corollary:

The subset of regular points is dense in $\operatorname{spt} Q$.

Knowing no counterexamples one is tempted to conjecture that the density hypothesis in the theorem could be replaced by the weaker hypothesis

$$
\Theta^{m}\left[\|Q\| L\left\{x: \Theta^{m}(\|Q\|, x)<\Theta^{* m}(\|Q\|, a)\right\}, a\right]=0,
$$

which holds for $\mathcal{H}^{m}$ almost all $a$ in spt $Q$. If this were true one could conclude that the singular subset of spt $Q$ has $\mathcal{H C}^{m}$ measure zero. For two special dimensions such results have been proved [23, $\$ 5.3 .20,5.3 .19]$ :

If $m=1$, then the singular subset of $\operatorname{spt} Q$ is empty.

If $m=n-1$, then the singular subset of spt $Q$ has $\mathcal{H C}^{m}$ measure zero.

Examples show that for $1<m<n-1$ the singular subset of spt $Q$ can have dimension $m-2$. For instance

$$
\mathbf{R}^{m+2} \cap\left\{x:\left(x_{1}=0 \text { and } x_{2}=0\right) \text { or }\left(x_{m+1}=0 \text { and } x_{m+2}=0\right)\right\}
$$

is the support of an area minimizing current in $\mathbf{R}^{m+2}$. One may still hope that $m-2$ is the highest possible dimension of the singular set of an $m$ dimensional locally rectifiable current with boundary zero in $A$ which minimizes an elliptic integrand of class 3 with respect to $\mathscr{Z}_{m}(A, \varnothing)$.

It was shown recently [5] that in case $m=n-1$ the singular subset of $\operatorname{spt} Q$ has $\mathcal{H}^{m-2}$ measure zero.

Even more recently Almgren announced [Notices Amer. Math. Soc. 24 (1977), A-541] that he has found a proof of the following proposition concerning the case when $\Psi$ is the area integrand of arbitrary degree $m$ on an open subset $A$ of $\mathbf{R}^{n}$ :

If $Q$ is an area minimizing locally rectifiable $m$ dimensional current in $A$, with $\partial Q=0$, then the singular subset of spt $Q$ has $\mathcal{H}^{m}$ measure zero.

Precise estimates of the maximal dimension of the singular set of a minimizing current have been obtained for the area integrand of degree $m$ on $\mathbf{R}^{m+1}$. Successive contributions by Fleming, De Giorgi, Triscari, Almgren, Simon and Federer led to the following results [23, \$5.4.15] and [25].

Suppose $A$ is an open subset of $\mathbf{R}^{m+1}$,

$$
Q \in \Re_{m}^{\text {loc }}(A), \quad \partial Q=0
$$

and $Q$ is area minimizing with respect to $\mathscr{Z}_{m}(A, \varnothing)$.

If $m<6$, then the singular subset of spt $Q$ is empty.

If $m>7$, then the Hausdorff dimension of the singular subset of spt $Q$ does not exceed $m-7$. 
Analogous propositions are true for the area integrand of degree $m$ induced by any Riemannian metric of class 3 on a manifold $A$ of dimension $m+1$.

Singular sets with dimension $m-7$ really occur. For instance, if $m \geqslant 7$, then

$$
\mathbf{R}^{m+1} \cap\left\{x: \sum_{i=1}^{4}\left(x_{i}\right)^{2}=\sum_{i=5}^{8}\left(x_{i}\right)^{2}\right\}
$$

is the support of an area minimizing $m$ dimensional locally rectifiable current with boundary zero in $\mathbf{R}^{m+1}$, as first shown by Bombieri, De Giorgi and Giusti [8]. Later we will sketch a simple proof using an appropriate differential form. Other examples were found by Lawson [52]. For $m>7$ it is still unknown whether the $m-7$ dimensional Hausdorff measure of the singular set must be locally finite in $A$.

Replacing integral flat chains by flat chains modulo $\nu$, one similarly studies minimizing chains with coefficients in the cyclic group $\mathbf{Z}_{\nu}=\mathbf{Z} / \nu \mathbf{Z}$. This change in coefficient group hardly affects the existence theory, but the modified interior structure theory exhibits some distinctly new features. The nature of the singularities of minimizing chains modulo $\nu$ really depends on the value of $\nu$.

In case $\nu=2$, the singular set of an area minimizing $m$ dimensional flat chain modulo 2 in any Riemannian manifold $A$ has Hausdorff dimension at most $m-2[25, \S 3]$. Moreover, the singular set is empty if $m=1$, and has dimension at most $m-7$ if $\operatorname{dim} A=m+1$.

In case $\nu=3$, the singular set of an area minimizing $m$ dimensional flat chain modulo 3 in any Riemannian manifold has Hausdorff dimension at most $m-1$. Singularities occur even if $m=1$; for example a length minimizing flat chain modulo 3 in $\mathbf{C}$ is supported by the union of the three line segments from zero to the cube roots of unity. According to J. E. Taylor [76] the singular set of any area minimizing 2 dimensional flat chain modulo 3 in $\mathbf{R}^{3}$ is the union of a locally finite family of smooth curves, along each of which three surfaces composed of regular points meet at $120^{\circ}$ angles.

Next we consider chains with real coefficients. For every positive convex integrand $\Psi$ the following theorem [27, 83.9] guarantees the existence of $\Psi$ minimizing real flat chains, with respect to suitable spaces of real flat cycles and boundaries.

If $K$ is a compact subset of $A$,

$$
\begin{aligned}
& \mathbf{Z}_{m, K}(A, B)=\mathbf{Z}_{m}(A, B) \cap\{R: \text { spt } R \subset K\}, \\
& \mathbf{B}_{m, K}(A, B)=\mathbf{B}_{m}(A, B) \cap\{R: \text { spt } R \subset K\}
\end{aligned}
$$

and $S \in \mathbf{F}_{m, K}(U)$, then there exist $m$ dimensional real flat chains $Q, Q^{\prime}$ with finite mass such that

$$
\begin{gathered}
Q-S \in \mathbf{Z}_{m, K}(A, B) \quad \text { and } \quad \Psi_{\mathbf{Z}_{m, K}(A, B)}(S)=\int_{Q} \Psi \\
Q^{\prime}-S \in \mathbf{B}_{m, K}(A, B) \text { and } \Psi_{\mathbf{B}_{m, K}(A, B)}(S)=\int_{Q^{\prime}} \Psi .
\end{gathered}
$$


We refer to $Q$ (or $Q^{\prime}$ ) as absolutely (or homologically) $\Psi$ minimizing in $K$ with respect to $(A, B)$ over $R$.

In general, minimizing real flat chains do not enjoy regularity properties analogous to those of minimizing rectifiable currents. We will soon illustrate this deficiency by an example. However, partial compensation is afforded by the fact that often the minima $\Psi_{\Gamma}(S)$ associated with sets $\Gamma$ of real flat chains can be alternately characterized by the values of $S$ on suitable sets of differential forms [27, $\$ 4.11(2), 4.10(4)]$.

If $S \in \mathbf{F}_{m, K}(U)$, then

$$
\begin{aligned}
& \Psi_{\mathbf{Z}_{m, K}(A, B)}(S)=\sup \{ S(d \psi): \psi \in \mathcal{E}^{m-1}(U), \text { spt } \psi \subset U \sim B, \\
&\left.\langle\xi, d \psi(x)\rangle \leqslant \Psi(x, \xi) \text { for }(x, \xi) \in K \times \wedge_{m} \mathbf{R}^{n}\right\}
\end{aligned}
$$

and

$$
\begin{array}{r}
\Psi_{\mathbf{B}_{m, K}(A, B)}(S)=\sup \left\{S(\phi): \phi \in \mathcal{E}^{m}(U), d \phi=0, \text { spt } \phi \subset U \sim B,\right. \\
\left.\langle\xi, \phi(x)\rangle \leqslant \Psi(x, \xi) \text { for }(x, \xi) \in K \times \wedge_{m} \mathbf{R}^{n}\right\} .
\end{array}
$$

In case $S \in \mathbf{F}_{m, K}(U) \cap \mathbf{M}_{m}(U)$ the equation

$$
\Psi_{\mathrm{B}_{m, K}(A, B)}(S)=\int_{S} \Psi
$$

holds whenever there exists a differential form $\phi$ which has the properties listed above and which also satisfies the condition

$$
\langle\vec{S}(x), \phi(x)\rangle=\Psi[x, \vec{S}(x)] \text { for }\|S\| \text { almost all } x .
$$

We proceed to the use of such differential forms in several examples.

(1) Given any nonnegative real valued $L^{1}$ measurable function $g$ such that

$$
\int_{C} g d \varrho^{1}<\infty \text { for every compact } C \subset \mathbf{R},
$$

we define $\zeta(x)=\left(0, g\left(x_{1}\right)\right) \in \mathbf{R}^{2}$ for $x \in \mathbf{R}^{2}$,

$$
\begin{gathered}
T=\mathscr{L}^{2} \wedge \zeta \in \mathbf{N}_{1}^{\mathrm{loc}}\left(\mathbf{R}^{2}\right) \quad \text { with } \partial T=0,\|T\|=\mathcal{L}^{2} L\left(g \circ X_{1}\right), \\
\vec{T}(x)=(0,1) \text { and }\left\langle\vec{T}(x), D X_{2}(x)\right\rangle=1 \text { for }\|T\| \text { almost all } x .
\end{gathered}
$$

For each compact $E \subset \mathbf{R}^{2}$ our sufficient conditions are satisfied by $S=T L E$, $\phi=D X_{2}$ and the length integrand $\Psi$, hence

$$
\Psi_{\mathrm{B}_{1}\left(\mathrm{R}^{2}, \varnothing\right)}\left(T\llcorner E)=\int_{T\llcorner E} \Psi .\right.
$$

Thus $T$ is length minimizing with respect to $\mathbf{R}^{2}$ over the coefficient group $\mathbf{R}$, but of course $T$ is only as smooth as the function $g$.

(2) On the space $\mathrm{C}^{n}$ with the usual coordinate functions $Z_{j}=X_{j}+Y_{j} \mathbf{i}$ we consider the differential forms

$$
\begin{gathered}
\Omega=\frac{\mathbf{i}}{2} \sum_{j=1}^{n} D Z_{j} \wedge D \bar{Z}_{j}=\sum_{j=1}^{n} D X_{j} \wedge D Y_{j} \in \mathcal{E}^{2}\left(\mathbf{C}^{n}\right), \\
\phi_{\kappa}=\Omega^{\kappa} / \kappa ! \in \mathcal{E}^{2 \kappa}\left(\mathbf{C}^{n}\right) \text { for } \kappa=1, \ldots, n,
\end{gathered}
$$


and recall Wirtinger's inequality $[23, \S 1.8 .2]$

$$
\left\langle\xi, \phi_{\kappa}(z)\right\rangle \leqslant\|\xi\| \text { for }(z, \xi) \in \mathbf{C}^{n} \times \wedge_{2 \kappa} \mathbf{C}^{n},
$$

in which equality holds if and only if $\xi$ belongs to the convex hull of

$$
\left\{v_{1} \wedge \mathbf{i} v_{1} \wedge \cdots \wedge v_{\kappa} \wedge \mathbf{i} v_{\kappa}: v_{1} \in \mathbf{C}^{n}, \ldots, v_{\kappa} \in \mathbf{C}^{n}\right\} .
$$

It follows that if $T$ is a complex $\kappa$ dimensional positive holomorphic chain in an open subset $U$ of $\mathbf{C}^{n}$, then

$$
\left\langle\vec{T}(z), \phi_{\kappa}(z)\right\rangle=1 \text { for }\|T\| \text { almost all } z,
$$

and for each compact $E \subset U$ our sufficient conditions are satisfied by $S=T L E, \phi=\phi_{\kappa}$ and the mass integrand $\Psi$, hence

$$
\Psi_{\mathrm{B}_{2 \times}(U, \varnothing)}(T L E)=\int_{T L E} \Psi .
$$

Thus $T$ is mass minimizing with respect to $U$ over the coefficient group $\mathbf{R}$.

One sees similarly that every complex $\kappa$ dimensional positive holomorphic chain in any Kaehler manifold $\boldsymbol{A}$ is mass minimizing with respect to $\mathbf{B}_{2 \kappa}(A, \varnothing)$.

(3) Suppose $W$ is an open subset of $\mathbf{R}^{m}$ and $f \in \mathcal{E}^{0}(W)$ satisfies the minimal surface equation

$$
\sum_{j=1}^{m} D_{j}\left[\left(1+\|D f\|^{2}\right)^{-1 / 2} D_{j} f\right]=0 .
$$

The function $f$ is the support of the current

$$
T=F_{\#}\left(\mathbf{E}^{m} L W\right) \in \mathbf{I}_{m}^{\text {loc }}(W \times \mathbf{R})
$$

where $F(w)=(w, f(w)) \in W \times \mathbf{R}$ for $w \in W$, and the unit vectorfield $\zeta$ defined by the formula

$$
\zeta(w, y)=\|D F(w)\|^{-1}(-\operatorname{grad} f(w), 1) \in \mathbf{R}^{m} \times \mathbf{R}
$$

for $(w, y) \in W \times \mathbf{R}$ has divergence zero. Using the standard coordinate functions $X_{1}, \ldots, X_{m+1}$ on $\mathbf{R}^{m} \times \mathbf{R} \cong \mathbf{R}^{m+1}$ we obtain the differential form

$$
\phi=\zeta\lrcorner\left(D X_{1} \wedge \cdots \wedge D X_{m+1}\right) \in \mathcal{E}^{m}(W \times \mathbf{R})
$$

with $d \phi=0,\|\phi\|=1$, and

$$
\langle\vec{T}(w, y), \phi(w, y)\rangle=1 \quad \text { for }(w, y) \in \operatorname{spt} T
$$

because $\left\langle D_{1} F(w) \wedge \cdots \wedge D_{m} F(w), \phi[F(w)]\right\rangle=J_{m} F(w)$ for $w \in W$. It follows that $T$ is area minimizing with respect to $\mathbf{B}_{m}(W \times \mathbf{R}, \varnothing)=\mathbf{Z}_{m}(W \times$ $\mathrm{R}, \varnothing)[23,85.4 .18]$.

In the interest of wider applicability it is essential that one can liberalize the sufficient conditions for minimality by requiring much less smoothness of the differential form $\phi$. A general theorem [27, §6.2] on flat cochains corresponding to differential forms with certain types of singularities has the following corollary.

If $Z$ is an open subset of $U, m<n-1$, 


$$
\begin{gathered}
\mathcal{H}^{m}(U \sim Z)=0, \quad \phi \in \mathcal{E}^{m}(Z), \quad d \phi=0, \\
\langle\xi, \phi(x)\rangle\left\langle\Psi(x, \xi) \quad \text { for }(x, \xi) \in Z \times \wedge_{m} \mathbf{R}^{n},\right. \\
T \in \mathbf{F}_{m}^{\text {loc }}(U), \quad T \text { is representable by integration, } \\
\langle\vec{T}(x), \phi(x)\rangle=\Psi[x, \vec{T}(x)] \text { for }\|T\| \text { almost all } x,
\end{gathered}
$$

then $T$ is $\Psi$ minimizing with respect to $\mathbf{B}_{m}(U, \varnothing)$.

We will give two examples. Both employ the angle forms

$$
\Upsilon_{\kappa} \in \mathcal{E}^{\kappa}\left(\mathbf{R}^{\kappa+1} \sim\{0\}\right)
$$

defined by the equation

$$
\left.\Upsilon_{\kappa}(x)=(-1)^{\kappa}|x|^{-\kappa-1} x\right\lrcorner\left(X_{1} \wedge \cdots \wedge X_{\kappa+1}\right)
$$

for $x \in \mathbf{R}^{\kappa+1} \sim\{0\}$, whence $\left\|\Upsilon_{\kappa}(x)\right\|=|x|^{-\kappa}$ and $d \Upsilon_{\kappa}(x)=0$.

(4) We use the proper embedding $F: \mathbf{R}^{2} \rightarrow \mathbf{R}^{3}$ such that

$$
\boldsymbol{F}(w)=\left(w_{2} \cos \left(w_{1}\right), w_{2} \sin \left(w_{1}\right), w_{1}\right) \text { for } w \in \mathbf{R}^{2}
$$

to construct the half helicoid

$$
T=F_{\#}\left(\mathbf{E}^{2} L\left\{w: w_{2} \geqslant 0\right\}\right) \in \mathbf{I}_{2}^{\mathrm{loc}}\left(\mathbf{R}^{3}\right),
$$

and note that $\partial T=G_{\#} \mathbf{E}^{1}$ with $G(s)=(0,0, s)$ for $s \in \mathbf{R}$. We further define

$$
\begin{gathered}
p(x)=\left(x_{1}, x_{2}\right) \text { and } r(x)=|p(x)| \text { for } x \in \mathbf{R}^{3}, \\
Z=\mathbf{R}^{3} \cap\{x: r(x)>0\}, \\
\phi=\left(r^{2}+1\right)^{-1 / 2}\left(r^{2} p^{\#} \Upsilon_{1}+D X_{3}\right) \wedge D r \in \mathcal{E}^{2}(Z) .
\end{gathered}
$$

Inasmuch as $\mathcal{H}^{2}\left(\mathbf{R}^{3} \sim Z\right)=0, d \phi=0,\|\phi\|=1$, and

$$
\langle\vec{T}(x), \phi(x)\rangle=1 \text { for }\|T\| \text { almost all } x
$$

because $\left\langle D_{1} F(w) \wedge D_{2} F(w), \phi[F(w)]\right\rangle=J_{2} F(w)$ if $w_{2}>0$, our corollary implies that $T$ is area minimizing with respect to $\mathbf{R}^{3}$ over $\mathbf{R}$. However, the whole helicoid $F_{\#} \mathbf{E}^{2}$ is not area minimizing $[\mathbf{6 2}, \S 111]$.

(5) In $U=\mathbf{R}^{4} \times \mathbf{R}^{4}$ we consider the current

$$
T=\partial\left[\left(\mathbf{E}^{4} \times \mathbf{E}^{4}\right)\llcorner U \cap\{(x, y):|x|<|y|\}] \in \mathbf{I}_{7}^{\text {loc }}(U)\right.
$$

with spt $T=U \cap\{(x, y):|x|=|y|\}$ and $\partial T=0$. For $(x, y) \in U$ we let

$$
p(x, y)=x, \quad q(x, y)=y, \quad r(x, y)=\left(|x|^{2}+|y|^{2}\right)^{1 / 2} ;
$$

in case $r(x, y)>0$ we define $\theta(x, y)$ so that $0 \leqslant \theta(x, y) \leqslant \pi / 2$ and

$$
|x|=r(x, y) \cos [\theta(x, y)], \quad|y|=r(x, y) \sin [\theta(x, y)] .
$$

The restrictions of $p, q, r, \theta$ to $Z=\left(\mathbf{R}^{4} \sim\{0\}\right) \times\left(\mathbf{R}^{4} \sim\{0\}\right)$ are of class $\infty$, and $\mathcal{H}^{7}(U \sim Z)=0$. We further let

$$
\begin{gathered}
u=\left(r^{7} / 7\right)[\sin (2 \theta)]^{49 / 8}, \\
\phi=\frac{1}{8} D u \wedge p^{\#} \Upsilon_{3} \wedge q^{\#} \Upsilon_{3} \in \mathcal{E}^{7}(Z) .
\end{gathered}
$$


After verifying through elementary computations [27, §6.3] that $d \phi=0$, $\|\phi(x, y)\| \leqslant 1$ for $(x, y) \in Z$, and

$$
\langle\vec{T}(x, y), \phi(x, y)\rangle=1 \quad \text { for }(x, y) \in Z \cap \operatorname{spt} T,
$$

one infers from our corollary that $T$ is area minimizing with respect to $U$ over R. The singular subset of spt $T$ consists of the point $(0,0)$.

For each integer $m>7$ the current

$$
T \times \mathbf{E}^{m-7} \in \mathbf{I}_{m}^{\text {loc }}\left(\mathbf{R}^{4} \times \mathbf{R}^{4} \times \mathbf{R}^{m-7}\right),
$$

which has boundary zero, is area minimizing with respect to $\mathbf{R}^{4} \times \mathbf{R}^{4} \times$ $\mathbf{R}^{m-7} \simeq \mathbf{R}^{m+1}$, and the singular subset of the support of this current equals $\{0\} \times\{0\} \times \mathbf{R}^{m-7}$.

Given an integral flat chain $S$, one may reasonably choose to minimize the integral of $\Psi$ over currents with finite mass which differ from $S$ by integral flat cycles, or one may allow the differences to be real flat cycles. Similarly one may admit either integral boundaries or real boundaries as differences. Of course the values of the minima are likely to depend, in general, on the choice of $\mathbf{Z}$ or $\mathbf{R}$ as coefficient group. We will now review the known results $[27, \S 5]$ comparing such minima. For this purpose we assume that $K$ is a compact subset of $A, K$ and $K \cap B$ are Lipschitz neighborhood retracts in $U$, and $K$ equals the union of a countable sequence of submanifolds $H_{i}$ of class 1 of $U$ such that $\Psi$ is $H_{i}$ flat [27, \$2.13]. The last hypothesis is quite tolerable, because for instance the mass integrand associated with any Riemannian metric on $U$ is $H$ flat for every submanifold $H$ of class 1 of $U$.

If $\mathbf{S} \in \mathscr{F}_{m, K}(U)$, then

$$
\Psi_{\mathbf{Z}_{m, K}(A, B)}(S)=\inf _{0<j \in \mathbf{Z}} j^{-1} \Psi_{\Psi_{m, K}(A, B)}(j S)
$$

and

$$
\Psi_{\mathbf{B}_{m, K}(A, B)}(S)=\inf _{0<j \in \mathbf{Z}} j^{-1} \Psi_{\mathscr{B}_{m, K}(A, B)}(j S) .
$$

(One may replace $\inf _{0<j \in \mathbf{Z}}$ by $\lim _{\mathbf{Z} \in j \rightarrow \infty}$.)

For example, if $K=A$ and

$$
\alpha \in \mathbf{H}_{m}(A, B ; \mathbf{Z}), \quad \beta \in \mathbf{H}_{m}(A, B ; \mathbf{R}) \quad \text { with } \alpha \subset \beta,
$$

one can take $S \in \alpha$ to obtain

$$
\inf \left\{\int_{Q} \Psi: Q \in \beta\right\}=\inf \left\{j^{-1} \int_{Q^{\prime}} \Psi: 0<j \in \mathbf{Z}, Q^{\prime} \in j \alpha\right\} .
$$

It has also been shown that

$$
\begin{aligned}
\Psi_{\mathbf{Z}_{m, K}(A, B)}(S) & =\Psi_{\mathscr{I}_{m, K}(A, B)}(S) \\
\text { in case } m & =1 \text { and } \Psi \text { is an even integrand, }
\end{aligned}
$$

but in case $m \geqslant 2$ the equation (I) often fails for the mass integrand $\Psi$ in a Riemannian manifold $A$. Furthermore

$$
\Psi_{\mathbf{B}_{m, K}(A, B)}(S)=\Psi_{\mathscr{S}_{m, K}(A, B)}(S)
$$

in case $A$ is an $m+1$ dimensional orientable manifold of class 1 and $\Psi$ is an even integrand, 
but in case $\operatorname{dim} A>m+1$ or $A$ is nonorientable the equation (II) often fails for the mass integrand.

In general one really needs to use infinitely many multiples $j S$, because it can happen that

$$
\Psi_{\mathbf{B}_{m, K}(A, B)}(S)<j^{-1} \Psi_{\mathscr{B}_{m, K}(A, B)}(j S)
$$

for every positive integer $j$. Such examples, with $\Psi=$ the mass integrand and $B=\varnothing$, have been constructed by Almgren [27, \$5.11] for $m=1$ in a Riemannian manifold $A$ diffeomorphic with $\mathbf{S}^{1} \times \mathbf{S}^{2}$, and by Lawson [53] for $m \geqslant 3$ in a $2 m$ dimensional flat torus $A$. Thus Lawson found a complex 4 dimensional Abelian variety with a 4 dimensional integral homology class $\alpha$ such that an invariant positive current of type $(2,2)$ belongs to the real homology class $\beta$ containing $\alpha$, but no positive holomorphic chain belongs to any integral multiple of $\alpha$.

We have seen that minimization over $\mathbf{Z}$ differs globally from minimization over R. However, it is not known whether these two properties differ locally. The theory of geodesic fields shows that they coincide in some neighborhood of every regular point.

11. Slicing. In conclusion we briefly discuss the measuretheoretic method $[23,84.3]$ of slicing an $m$ dimensional flat chain

$$
S \in \mathbf{F}_{m}(U)
$$

by a locally Lipschitzian map

$$
f: U \rightarrow \mathbf{R}^{\mu},
$$

where $m \geqslant \mu$. Corresponding to $y \in \mathbf{R}^{\mu}$ one frequently needs to consider the part of $S$ in $f^{-1}\{y\}$ as an $m-\mu$ dimensional current

$$
\langle S, f, y\rangle \in \mathscr{D}_{m-\mu}(U) \text {. }
$$

For the definition of this concept we will need the volume form

$$
\Omega=D Y_{1} \wedge \cdots \wedge D Y_{\mu} \in \mathcal{E}^{\mu}\left(\mathbf{R}^{\mu}\right),
$$

where $Y_{1}, \ldots, Y_{\mu}$ are the standard coordinate functions on $\mathrm{R}^{\mu}$.

Our general procedure can be motivated by the special case when $S=$ $\left(\mathcal{F C}^{m} L W\right) \wedge \xi$ for some $m$ dimensional submanifold $W$ of class 1 of $U$ such that $\mathcal{F \mathcal { H }}^{m}(W)<\infty$ and $W$ is oriented by the $m$ vectorfield $\xi$. For $x \in W$ we factor

$$
\xi(x)=\eta(x) \wedge \zeta(x)
$$

so that $\eta(x)$ is a simple $\mu$ vector of $\operatorname{Tan}(W, x)$,

$$
\|\eta(x)\|=1 \text { and }\left\langle\eta(x), f^{\#} \Omega(x)\right\rangle=J_{\mu}(f \mid W)(x) .
$$

$\zeta(x)$ is a simple $m-\mu$ vector of $\operatorname{Tan}(W, x)$,

$$
\|\zeta(x)\|=1 \text { and } \operatorname{ker} D(f \mid W)(x) \text { is associated with } \zeta(x)
$$

in case $J_{\mu}(f \mid W)(x)>0$. Using the coarea formula we find that if $\psi \in$ $\mathscr{D}^{m-\mu}(U)$ and $\phi$ is any real valued bounded Baire function on $\mathbf{R}^{\mu}$, then 


$$
\begin{aligned}
& {\left[S\left\llcorner f^{\#}(\phi \Omega)\right](\psi)=S\left[f^{\#}(\phi \Omega) \wedge \psi\right]\right.} \\
& =\int_{W}\left\langle\xi(x), f^{\#}(\phi \Omega)(x) \wedge \psi(x)\right\rangle d \mathcal{H}^{m} x \\
& =\int_{W} \phi[f(x)] J_{\mu}(f \mid W)(x)\langle\zeta(x), \psi(x)\rangle d \mathcal{H}^{m} x \\
& =\int_{\mathbf{R}^{\mu}} \phi(y) \int_{W \cap f^{-1}\{y\}}\langle\zeta(x), \psi(x)\rangle d \mathcal{H}^{m-\mu} x d \mathcal{L}^{\mu} y \\
& =\int_{\mathbf{R}^{\mu}} \phi(y)\left[\left(\mathcal{H}^{m-\mu}\left\llcorner W \cap f^{-1}\{y\}\right) \wedge \zeta\right](\psi) d \mathcal{L}^{\mu} y .\right.
\end{aligned}
$$

It follows from differentiation theory that, for $\mathfrak{L}^{\mu}$ almost all $y$,

$$
S\left\llcorner f^{\#}\left(b_{y, \rho} \Omega\right) /\left[\alpha(n) \rho^{n}\right] \rightarrow\left(\mathcal{H}^{m-\mu}\left\llcorner W \cap f^{-1}\{y\}\right) \wedge \zeta\right.\right.
$$

in $\mathscr{D}_{m-\mu}(U)$ as $\rho \downarrow 0$, where $b_{y, \rho}$ is the characteristic function of $\mathbf{B}(y, \rho)$.

When $S$ is an arbitrary flat chain one can still prove the existence of the slice

$$
\langle S, f, y\rangle=\lim _{\rho \downarrow 0} S L f^{\#}\left(b_{y, \rho} \Omega\right) /\left[\alpha(n) \rho^{n}\right] \in \mathscr{D}_{m-\mu}(U)
$$

for $\mathfrak{L}^{\mu}$ almost all $y$, with

$$
\begin{gathered}
\operatorname{spt}\langle S, f, y\rangle \subset f^{-1}\{y\} \cap \text { spt } S, \\
\partial\langle S, f, y\rangle=(-1)^{\mu}\langle\partial S, f, y\rangle \text { in case } m>\mu .
\end{gathered}
$$

Furthermore $\langle S, f, y\rangle \in \mathbf{F}_{m-\mu}(U)$ for $\mathcal{L}^{\mu}$ almost all $y$,

$$
\begin{gathered}
{\left[S\left\llcorner f^{\#} \Omega\right](\psi)=\int\langle S, f, y\rangle(\psi) d \mathcal{L}^{\mu} y \quad \text { for } \psi \in \mathscr{D}^{m-\mu}(U),\right.} \\
\mathbf{M}\left(S\left\llcorner f^{\#} \Omega\right)=\int \mathbf{M}\langle S, f, y\rangle d \mathcal{L}^{\mu} y .\right.
\end{gathered}
$$

In case $S$ is a rectifiable current, so is $\langle S, f, y\rangle$ for $\mathcal{L}^{\mu}$ almost all $y$.

In case $S \in \mathbf{N}_{m}(U)$ it is further known [26, \$3] that the condition

$$
\int|f(x)-y|^{1-\mu} d(\|S\|+\|\partial S\|) x<\infty
$$

implies that $\langle S, f, y\rangle$ exists, and that

$$
\langle S, f, y\rangle=(\partial S)\left\llcorner f^{\#} \Gamma_{y}+(-1)^{\mu} \partial\left(S\left\llcorner f^{\#} \Gamma_{y}\right)\right.\right.
$$

if either $f$ is of class 1 or $\mu=1$, where $\Gamma_{y}$ is the differential form of degree $\mu-1$ on $\mathbf{R}^{\mu}$ given by the formulae

$$
\left.\Gamma_{y}(z)=\mu^{-1} \alpha(\mu)^{-1}(-1)^{\mu-1}|z-y|^{-\mu}(z-y)\right\lrcorner\left(Y_{1} \wedge \cdots \wedge Y_{\mu}\right)
$$

for $z \in \mathbf{R}^{\mu} \sim\{y\}$, and $\Gamma_{y}(y)=0$. Moreover the set of those points $y$ in $\mathbf{R}^{\mu}$, for which the above condition fails, has $\mu-1$ dimensional potentialtheoretic capacity zero, hence Hausdorff dimension at most $\mu-1$.

In case $S$ is an analytic chain and $f$ is an analytic map it was proved by Hardt $[38,84.3]$ that $\langle S, f, y\rangle$ exists and is an analytic chain whenever $\operatorname{dim}\left(f^{-1}\{y\} \cap\right.$ spt $\left.S\right)<m-\mu$ and $\operatorname{dim}\left(f^{-1}\{y\} \cap\right.$ spt $\left.\partial S\right)<m-1-\mu$, 
and that $\langle S, f, y\rangle$ varies continuously on the set of all such points $y$. This makes slicing a useful method of assigning orientations and multiplicities to the varieties of solutions of analytic equations depending on parameters.

Among the applications of slicing are various integralgeometric formulae, such as

$$
\mathbf{M}(S)=c \int_{\mathbf{O}^{*}(n, \mu)} \int_{\mathbf{R}^{\mu}} \mathbf{M}\langle S, p, y\rangle d \mathcal{L}^{\mu} y d \theta_{n, \mu}^{*} p
$$

for $S \in \mathbf{F}_{m}\left(\mathbf{R}^{n}\right)$, where

$$
c=\frac{\Gamma[(m+1) / 2] \Gamma[(n-\mu+1) / 2]}{\Gamma[(n+1) / 2] \Gamma[(m-\mu+1) / 2]} .
$$

The current $\langle S, p, y\rangle$ should be viewed as the part of $S$ in the $n-\mu$ dimensional affine space $p^{-1}\{y\}$. Brothers has obtained general results of this type for homogeneous spaces of Lie groups, assuming only that the isotropy group acts with sufficient transitivity on the exterior algebra of the tangent space at the identity.

We use the subtraction map

$$
f: \mathbf{R}^{n} \times \mathbf{R}^{n} \rightarrow \mathbf{R}^{n}, \quad f(x, y)=x-y \quad \text { for }(x, y) \in \mathbf{R}^{n} \times \mathbf{R}^{n},
$$

and the diagonal map

$$
g: \mathbf{R}^{n} \rightarrow \mathbf{R}^{n} \times \mathbf{R}^{n}, g(x)=(x, x) \text { for } x \in \mathbf{R}^{n},
$$

whenever

$$
\begin{gathered}
S \in \mathbf{F}_{i}\left(\mathbf{R}^{n}\right), \quad T \in \mathbf{F}_{j}\left(\mathbf{R}^{n}\right), \quad S \times T \in \mathbf{F}_{i+j}\left(\mathbf{R}^{n} \times \mathbf{R}^{n}\right), \\
i+j \geqslant n \text { and }\langle S \times T, f, 0\rangle \in \mathbf{F}_{i+j-n}\left(\mathbf{R}^{n} \times \mathbf{R}^{n}\right)
\end{gathered}
$$

to define $[23,4.3 .20]$ the intersection current

$$
S \cap T \in \mathbf{F}_{i+j-n}\left(\mathbf{R}^{n}\right)
$$

characterized by the equation

$$
8_{\#}(S \cap T)=(-1)^{(n-i) j}\langle S \times T, f, 0\rangle .
$$

This operation generalizes alternating multiplication in the sense that

$$
\left(\mathbf{E}^{n} L \phi\right) \cap\left(\mathbf{E}^{n} L \psi\right)=\mathbf{E}^{n} L(\phi \wedge \psi)
$$

for $\phi \in \mathscr{Q}^{n-i}\left(\mathbf{R}^{n}\right)$ and $\psi \in \mathscr{Q}^{n-j}\left(\mathbf{R}^{n}\right)$.

The work of Hardt shows that $S \cap T$ exists whenever $S$ and $T$ are $i$ and $j$ dimensional analytic chains in $\mathbf{R}^{n}$ satisfying the two conditions

$$
\begin{gathered}
\operatorname{dim}(\text { spt } S \cap \operatorname{spt} T)<i+j-n, \\
\operatorname{dim}[(\text { spt } S \cap \operatorname{spt} \partial T) \cup(\text { spt } T \cap \operatorname{spt} \partial S)]<i+j-1-n .
\end{gathered}
$$

Moreover Hardt extended this intersection theory to analytic chains in arbitrary oriented analytic manifolds, and proved that the product has all the formal properties [38, 85.8] required for use in algebraic geometry and topology. He also proved the uniqueness [38, \$5.11] of such an intersection product. Thus slicing provides an analytic approach to intersection theory, which gives the same result as the customary method by way of homological 
algebra, and which sheds additional light on the continuous geometry.

Finally I want to mention that interesting applications of slicing to complex analysis have been made by King, Harvey, Lawson and Shiffman [44], [46], [47], [50].

\section{REFERENCES}

The following list is a supplement to the extensive bibliography in [23].

1. W. K. Allard, On the first variation of a varifold, Ann. of Math. (2) 95 (1972), 417-491.

2. , On the first variation of a varifold: Boundary behavior, Ann. of Math. (2) 101 (1975), 418-446.

3. W. K. Allard and F. J. Almgren, Jr., The structure of stationary one dimensional varifolds with positive density, Invent. Math. (to appear).

4. F. J. Almgren, Jr., Existence and regularity almost everywhere of solutions to elliptic variational problems with constraints, Mem. Amer. Math. Soc. No. 165 (1976).

5. F. J. Almgren, Jr., R. Schoen and L. Simon, Regularity and singularity estimates on hypersurfaces minimizing parametric elliptic variational integrals, Acta Math. (to appear).

6. F. J. Almgren, Jr. and W. P. Thurston, Examples of unknotted curves which bound only surfaces of high genus within their convex hulls, Ann. of Math. (2) 105 (1977), 527-538.

7. T. Bagby and W. P. Ziemer, Pointwise differentiability and absolute continuity, Trans. Amer. Math. Soc. 191 (1974), 129-148.

8. E. Bombieri, E. De Giorgi and E. Giusti, Minimal cones and the Bernstein theorem, Invent. Math. 7 (1969), 243-269.

9. E. Bombieri and E. Giusti, Harnack's inequality for elliptic differential equations on minimal surfaces, Invent. Math. 15 (1972), $24-46$.

10. K. E. Brakke, The motion of a surface by its mean curvature, Princeton Ph. D. Thesis, 1975.

11. J. E. Brothers, A characterization of integral currents, Trans. Amer. Math. Soc. 150 (1970), 301-325.

12. __ Stokes' theorem. I, II, Amer. J. Math. 62 (1970), 479-484; ibid. 63 (1971), 479-484.

13. __ Existence and structure of tangent cones at the boundary of an area minimizing integral current, Indiana Univ. Math. J. 26 (1977),

14. dimensional hemisphere, J. Differential Geometry 11 (1976), 387-400.

15. R. O. Davies, Increasing sequences of sets and Hausdorff measure, Proc. London Math. Soc. (3) 20 (1970), 222-236.

16. R. O. Davies and C. A. Rogers, The problem of subsets of finite positive measure, Bull. London Math. Soc. 1 (1969), 47-54.

17. E. De Giorgi, F. Colombini and L. C. Piccinini, Frontiere orientate di misura minima e questioni collegate, Pubbl. Classe Sci. Scuola Norm. Sup., Pisa, 1972.

18. L. R. Ernst, $A$ proof that $\mathcal{C}^{2}$ and $\mathcal{G}^{2}$ are distinct measures, Trans. Amer. Math. Soc. 173 (1972), 501-508.

19.,$A$ proof that $\mathcal{T}^{2}$ and $\mathfrak{I}^{2}$ are distinct measures, Trans. Amer. Math. Soc. 191 (1974), 363-372.

20. __ , T measure of Cartesian product sets, Proc. Amer. Math. Soc. 49 (1975), 199-202.

21. __ I T measure of Cartesian product sets. II, Trans. Amer. Math. Soc. 222 (1976), 211-220.

22. L. R. Ernst and G. Freilich, A Hausdorff measure inequality, Trans. Amer. Math. Soc. 219 (1976), 361-368.

23. H. Federer, Geometric measure theory, Grundlehren math. Wiss., Band 153, SpringerVerlag, Berlin and New York, 1969.

24. __ On spherical summation of the Fourier transform of a distribution whose partial derivatives are representable by integration, Ann. of Math. (2) 91 (1970), 136-143.

25. __ The singular sets of area minimizing rectifiable currents with codimension one and of area minimizing flat chains modulo two with arbitrary codimension, Bull. Amer. Math. Soc. 76 (1970), 767-771.

26. , Slices and potentials, Indiana Univ. Math. J. 21 (1971), 373-382. 
27. (1974), 351-407.

28. A minimizing property of extremal submanifolds, Arch. Rational Mech. Anal. 59 (1975), 207-217.

29. H. Federer and W. P. Ziemer, The Lebesgue set of a function whose distribution derivatives are pth power summable, Indiana Univ. Math. J. 22 (1972), 139-158.

30. A. T. Fomenko, Minimal compacta in Riemannian manifolds and Reifenberg's conjecture, Math. USSR Izv. 6 (1972), 1037-1066.

31. The multidimensional Plateau problem in Riemannian manifolds, Math. USSR Sbornik 18 (1972), 487-527.

32. R. Gariepy, Current valued measures and Geocze area, Trans. Amer. Math. Soc. 166 (1972), $133-146$.

33. 469-474.

34. __ Geometric properties of Sobolev mappings, Pacific J. Math. (to appear).

35. E. Giusti, Superfici cartesiani di area minima, Rend. Sem. Mat. Fis. Milano 40 (1970), 135-153.

36. C. Goffman, An example in surface area, J. Math. Mech. 19 (1969), 321-326.

37. C. Goffman and W. P. Ziemer, Higher dimensional mappings for which the area formula holds, Ann. of Math. (2) 93 (1970), 482-488.

38. R. M. Hardt, Slicing and intersection theory for chains associated with real analytic varieties, Acta Math. 129 (1972), 75-136.

39. Slicing and intersection theory for chains modulo $\nu$ associated with real analytic varieties, Trans. Amer. Math. Soc. 183 (1973), 327-340.

40. Homology theory for real analytic and semianalytic sets, Ann. Scuola Norm. Sup. Pisa (4) 2 (1975), 107-148.

41. __ Topological properties of subanalytic sets, Trans. Amer. Math. Soc. 211 (1975), 57-70.

42. (1976), 65-72.

43. Uniqueness of nomparametric area minimizing currents, Indiana Univ. Math. J. 26 On boundary regularity for integral currents or flat chains modulo two minimizing the integral of an elliptic integrand, Comm. Partial Differential Equations (to appear).

44. R. Harvey, Holomorphic chains and their boundaries, Proc. Sympos. Pure Math., vol. 30 Amer. Math. Soc., Providence, R. I., 1977, pp. 309-382.

45. R. Harvey and H. B. Lawson, Extending minimal varieties, Invent. Math. 28 (1975), 209-226.

46. _ On boundaries of complex analytic varieties, Ann. of Math. (2) 102 (1975), 223-290.

47. R. Harvey and B. Shiffman, A characterization of holomorphic chains, Ann. of Math. (2) 99 (1974), 553-587.

48. S. Kar, The $(\phi, 1)$ rectifiable subsets of Euclidean space, Indiana Ph.D. Thesis, 1975.

49. R. Kaufman and P. Mattila, Hausdorff dimension and exceptional sets of linear transformations, Ann. Acad. Sci. Fenn. Ser. AI 1 (1975), 387-392.

50. J. R. King, The currents defined by analytic varieties, Acta Math. 127 (1971), 185-220.

51. R. V. Kohn, An example concerning approximate differentiation, Indiana Univ. Math. J. 26 (1977), 393-397.

52. H. B. Lawson, Jr., The equivariant Plateau problem and interior regularity, Trans. Amer. Math. Soc. 173 (1973), 231-249.

53. __ The stable homology of a flat torus, Math. Scand. 36 (1975), 49-73.

54. H. B. Lawson, Jr. and R. Osserman, Nonexistence, nonuniqueness and irregularity of solutions to the minimal surface system, Acta Math. (to appear).

55. H. B. Lawson, Jr. and J. Simons, On stable currents and their applications to global problems in real and complex geometry, Ann. of Math. (2) 98 (1973), 427-450.

56. P. Mattila, Hausdorff $m$ regular and rectifiable sets in $n$ space, Trans. Amer. Math. Soc. 205 (1975), 263-274.

57. _ Hausdorff dimension, orthogonal projections and intersections with planes, Ann. Acad. Sci. Fenn. Ser. AI 1 (1975), 227-244.

58. N. G. Meyers and W. P. Ziemer, Integral inequalities of Poincaré and Wirtinger type, Amer. J. Math. (to appear). 
59. M. Miranda, Un principio di massimo forte per le frontiere minimale e una sua applicazione alla risoluzione del problema al contorno per l'equazione delle superfici di area minima, Rend. Sem. Mat. Univ. Padova 45 (1971), 355-366.

60. F. Morgan, $A$ smooth curve in $\mathbf{R}^{4}$ bounding a continuum of area minimizing surfaces, Duke Math. J. 43 (1976), 867-870.

61. Almost every smooth curve in $\mathbf{R}^{3}$ bounds a unique area minimizing surface, Princeton

Ph.D. Thesis, 1977.

62. J. C. C. Nitsche, Vorlesungen über Minimalflächen, Grundlehren math. Wiss., Band 199, Springer-Verlag, Berlin and New York, 1975.

63. R. Osserman, Minimal varieties, Bull. Amer. Math. Soc. 75 (1969), 1092-1120.

64. H. R. Parks, Some new constructions and estimates in the problem of least area, Trans. Amer. Math. Soc. (to appear).

65. __ A method for computing non-parametric area minimizing surfaces over $n$ dimensional domains, together with a priori error estimates, Indiana Univ. Math. J. 26 (1977), 625-643.

66. __ Explicit determination of area minimizing hypersurfaces, Duke Math. J. 44 (1977), 519-534.

67. S. O. Paur, Stokes' theorem for integral currents modulo v, Amer. J. Math. (to appear).

68. , An estimate of the density at the boundary of an integral current modulo $\nu$,

69. J. T. Pitts, Existence and regularity of minimal surfaces on Riemannian manifolds, Bull. Amer. Math. Soc. 82 (1976), 503-504.

70. C. A. Rogers, Hausdorff measures, Cambridge Univ. Press, London-New York, 1970.

71. E. Santi, Sul problema al contorno per l'equazione delle superfici di area minima su domini limitati qualunque, Annali dell'Universita di Ferrara 17 (1971), 13-26.

72. V. Scheffer, Regularity and irregularity of solutions to nonlinear second order elliptic systems of partial differential equations and inequalities, Princeton $\mathrm{Ph} . \mathrm{D}$. Thesis, 1974.

73. B. Shiffman, Applications of geometric measure theory to value distribution theory for meromorphic maps, Proc. Tulane Conf. on Value Distribution Theory, Part A, Dekker, New York, 1974, pp. 63-95.

74. L. Simon, Boundary regularity for solutions of the nonparametric least area problem, Ann. of Math. (2) 103 (1976), 429-455.

75. K. Steffen, Isoperimetrische Ungleichungen und das Plateausche Problem, Sonderforschungsbereich 40 (1973), Theoretische Mathematik, Universität Bonn.

76. J. E. Taylor, Regularity of the singular sets of two-dimensional area-minimizing flat chains modulo 3 in $\mathbf{R}^{3}$, Invent. Math. 22 (1973), 119-159.

77. __ The structure of singularities in soap-bubble-like and soap-film-like minimal surfaces, Ann. of Math. (2) 103 (1976), 489-539.

78. The structure of singularities in solutions to ellipsoidal variational problems with constraints in $\mathbf{R}^{3}$, Ann. of Math. (2) 103 (1976), 541-546.

79. , Boundary regularity for solutions to various capillarity and free boundary problems, Comm. Partial Differential Equations 2 (1977), 323-358.

80. A. I. Volpert, The space BV and quasilinear equations, Math. USSR Sbornik 2 (1967), 225-267.

81. W. P. Ziemer, Change of variables for absolutely continuous functions, Duke Math. J. 36 (1969), 171-178.

82. , Extremal length as a capacity, Michigan Math. J. 17 (1970), 117-128.

83. __ Slices of maps and Lebesgue area, Trans. Amer. Math. Soc. 164 (1972), 139-151.

84. __ Some remarks on harmonic measure in space, Pacific J. Math. 55 (1974), 629-637.

Department of Mathematics, Brown University, Providence, Rhode Island 02912 